\title{
Discrete Viewpoint Control to Reduce Cybersickness in Virtual Environments
}

\author{
by \\ Yasin Farmani
}

A thesis submitted to the Faculty of Graduate and Postdoctoral Affairs in partial fulfillment of the requirements for the degree of

Master of Computer Science

in

Human Computer Interaction

Carleton University

Ottawa, Ontario

(C) 2018, Yasin Farmani 


\begin{abstract}
Cybersickness in virtual reality (VR) is an on-going problem, despite recent advances in head-mounted displays (HMDs). In this thesis, we propose and evaluate a method for reducing the onset of cybersickness caused by illusions of self-motion (vection), when using stationary VR setups. Discrete viewpoint control techniques have been recently used by some VR developers and rely on reducing optic flow via inconsistent displacement. We propose two different techniques based on discrete movements in translational and rotational viewpoint movements. We ran two different user studies and measure participant cybersickness levels via the widely used Simulator Sickness Questionnaire (SSQ), as well as user reported levels of nausea, presence, and objective error rates. Overall, our results indicate that both viewpoints snapping and translation snapping significantly reduced SSQreported cybersickness levels by $40 \%$ for rotational viewpoint movement, and $50 \%$ for translational viewpoint movement. Both techniques resulted in a reduction in participant nausea levels, especially with longer VR exposure. Presence levels, error rate, and performance were not significantly different when using viewpoint snapping, or translation snapping as compared to a control condition with continuous viewpoint motion.
\end{abstract}




\section{Acknowledgments}

This is a genuine pleasure to express my deepest thanks to my mentor and supervisor Dr. Robert Teather from the School of Information Technology. This thesis would not be possible without his spectacular and brilliant guides and his financial and spiritual help. I extremely grateful to have advisors like Dr. Teather who accept my supervision and really improve my research skills beyond my expectations and imagination. I appreciate his ability to understand me as an international student. Thank for endeavoring my English Level, my writings and all the editing process with the patient.

I would like to say thanks to all my colleagues and classmates Siqi Lou, Adrian Ramcharitar, and Heather Qian. Thank you for all fun moments and memories that we created for each other. Thank you, Eduardo Soto, for your superior coding expertise and your help for creating my gaming software application. I owe a deep sense of gratitude to Matthew Thomas and his colleagues from Sinewave company in Ottawa. I would like to say thank you for their keen interest in every stage of my research with their suggestion with kindness and enthusiasm which help me to complete this thesis.

At the End, I want to say my deepest thanks to my parents and my lovely wife for their support and encouragement. Without their support, it was impossible to finish my higher education level. I never forget their kindness, patient, and support during my education. To my family, particularly my wife, thank you for your love and unwavering belief in me. Without you, I would not be the person I am today. 


\section{Table of Contents}

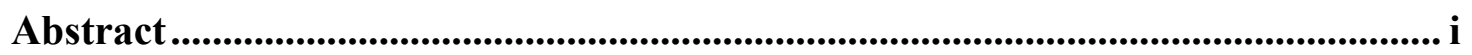

Acknowledgments ..................................................................................................... ii

Table of Contents ................................................................................................ iii

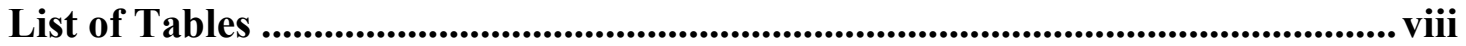

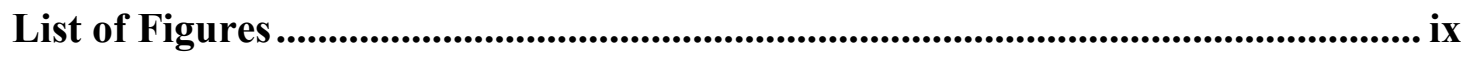

List of Appendices ................................................................................................................ xii

1 Chapter: Introduction .............................................................................................. 1

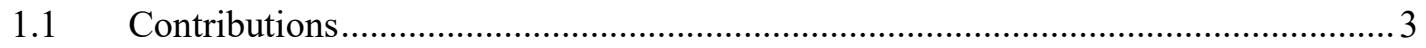

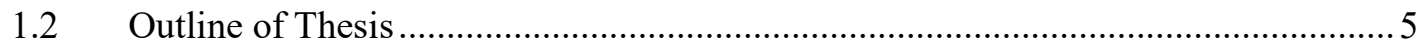

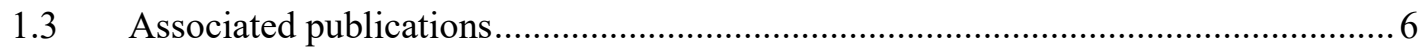

2 Chapter: Related work ........................................................................................... 7

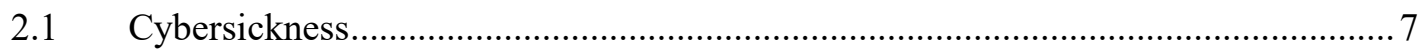

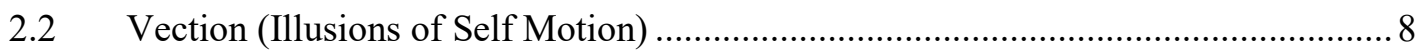

2.2.1 The Relationship Between Vection and Cybersickness....................................... 9

2.2.2 The relationship between vection speed and cybersickness ..............................9

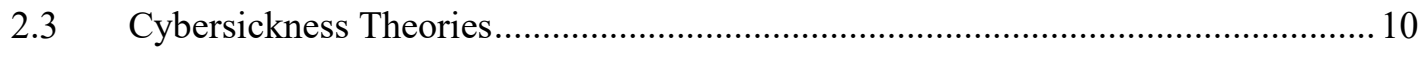

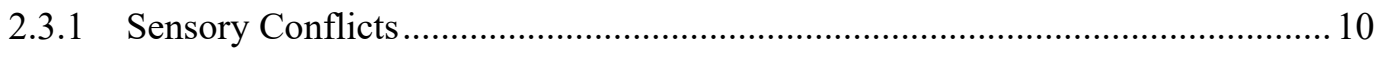

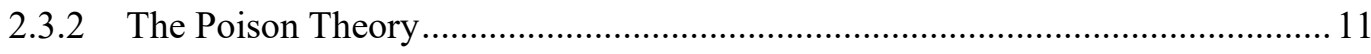

2.3.3 The Postural Instability Theory ................................................................. 12 
2.4 Contributing Factors to Cybersickness in VR

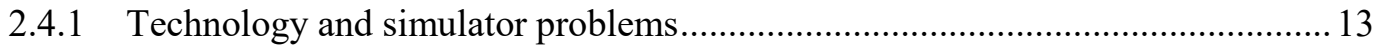

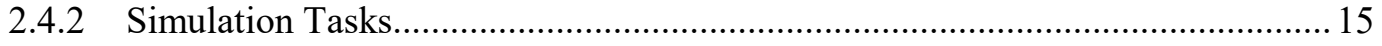

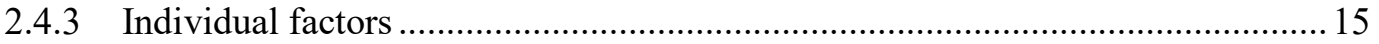

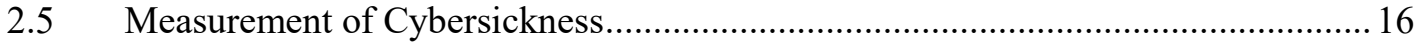

2.5.1 Subjective Measurement of Cybersickness ….................................................. 16

2.5.2 Objective Measurement of Cybersickness ....................................................... 18

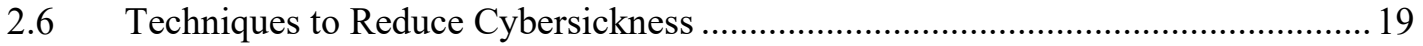

3 Chapter: Preliminary Studies, and Implementation of Techniques ............... 23

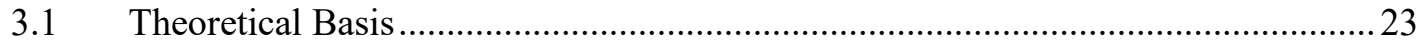

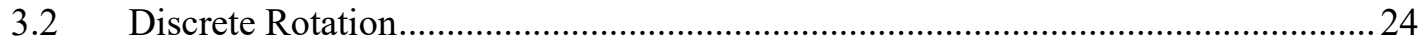

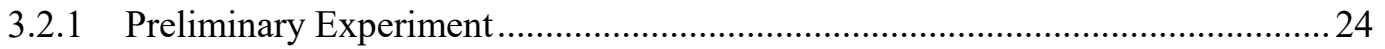

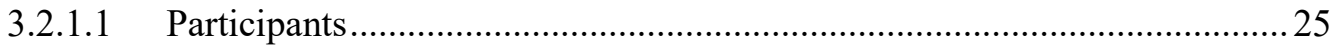

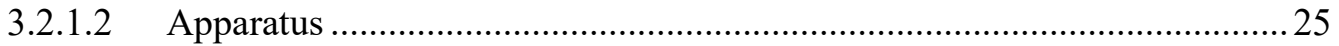

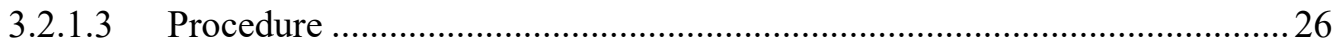

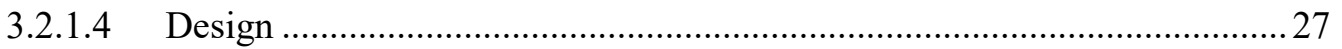

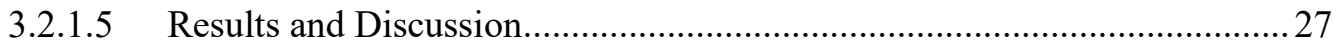

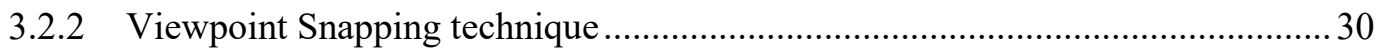

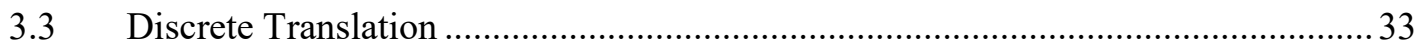

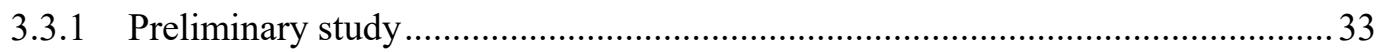




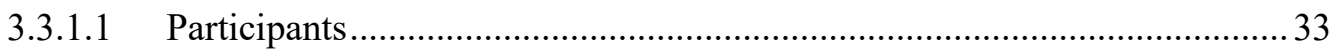

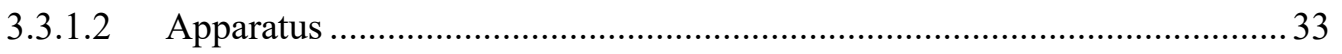

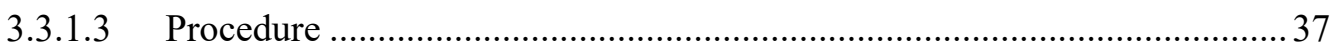

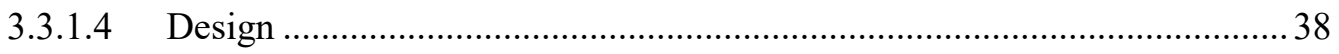

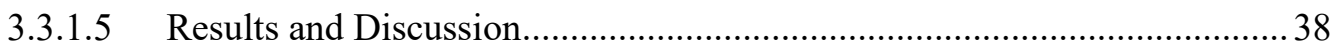

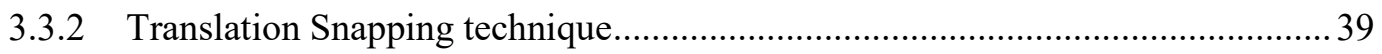

\section{Chapter: Viewpoint Snapping: User Study 1 ...................................................... 41}

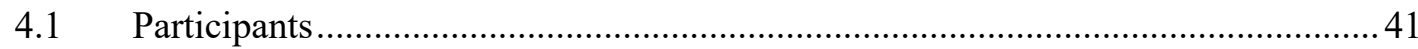

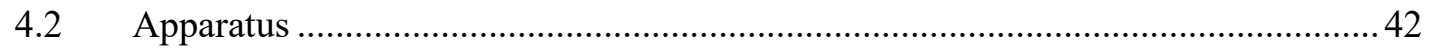

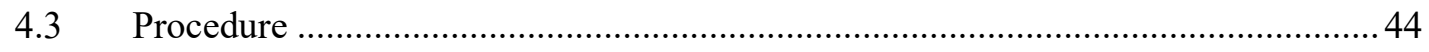

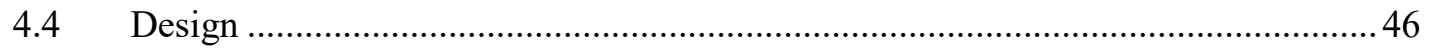

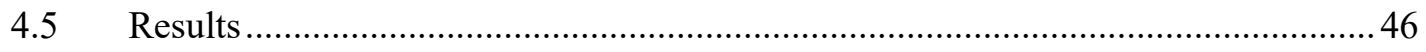

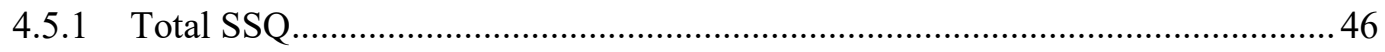

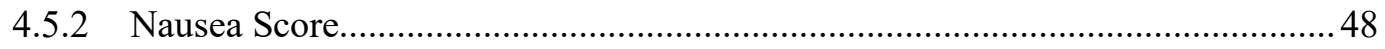

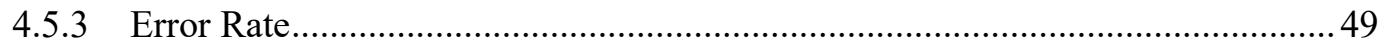

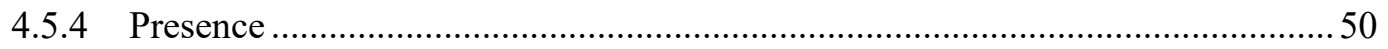

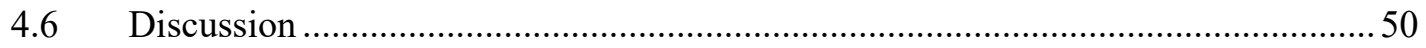

5 Chapter: Translation Snapping: User Study 2 ................................................ 53

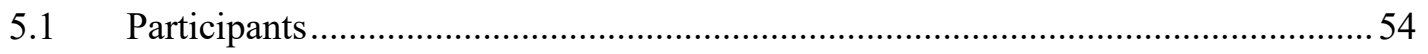

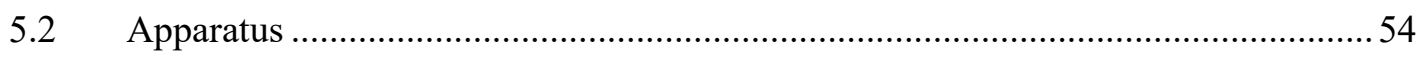


5.2.1 Hardware

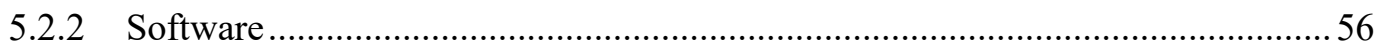

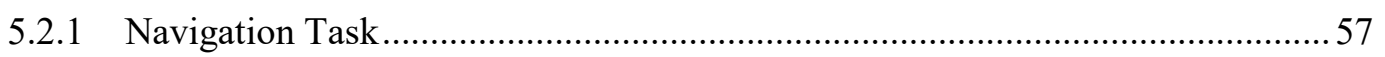

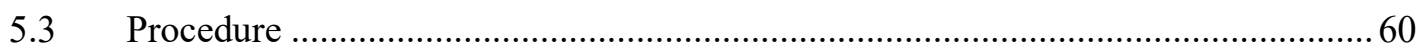

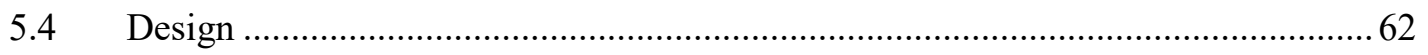

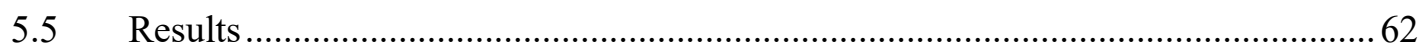

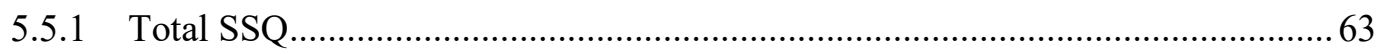

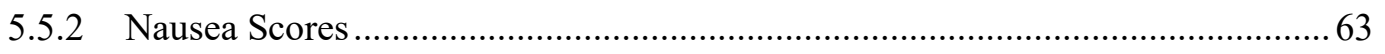

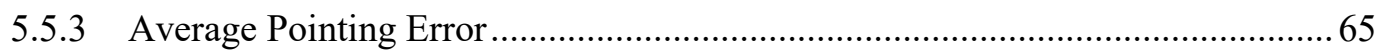

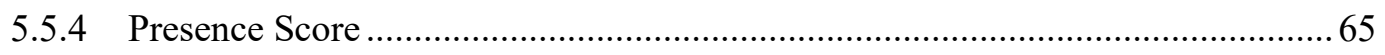

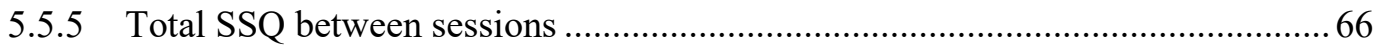

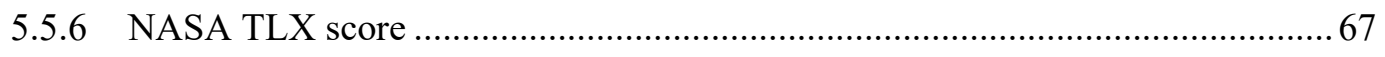

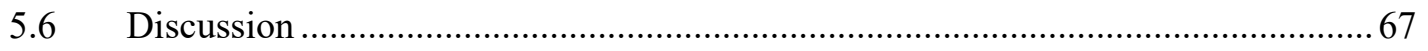

6 Chapter: Conclusion ........................................................................................ 70

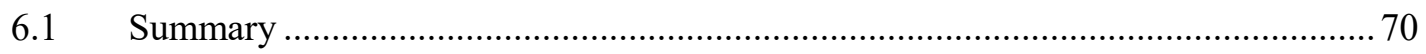

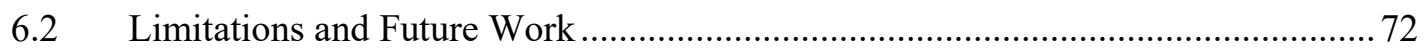

Appendices ..................................................................................................................... 74

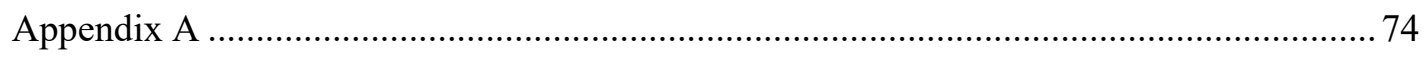

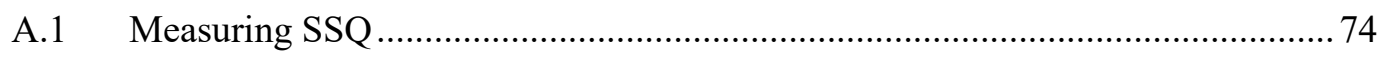

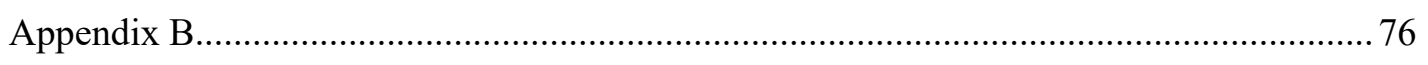


B.1 Consent Form for Viewpoint Snapping Study .............................................. 76

B.2 Consent form for Translation Snapping technique …......................................... 78

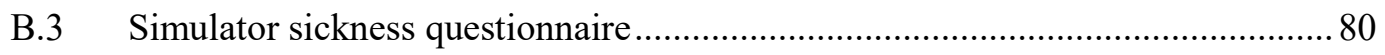

B.4 Witmer and Singer Presence questionnaire .................................................. 81

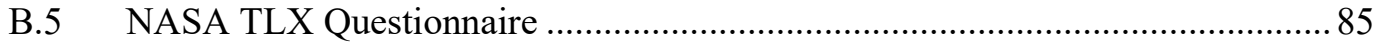

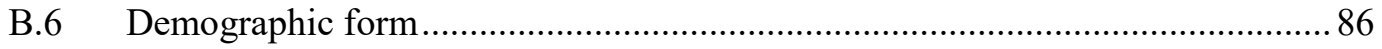

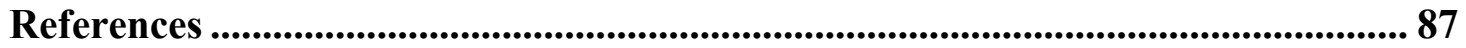




\section{List of Tables}

Table 1- Factor categories contributing to cybersickness [11] ................................ 13

Table 2- Example of Symptoms Clusters............................................................. 17

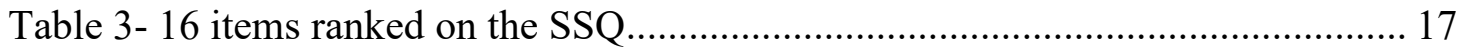

Table 4 - Experiment one - nausea scores by rotational speed. The red square indicates users withdraw from the study. It usually happens at the speed more than $65.3^{\circ} / \mathrm{s}$. Three users withdraw at speed of $100 \%$ s and two at speed of $200 \%$ s. These are depicted as red

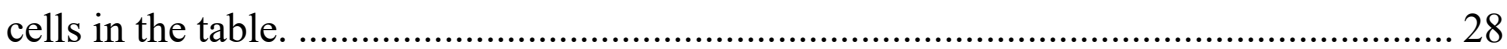

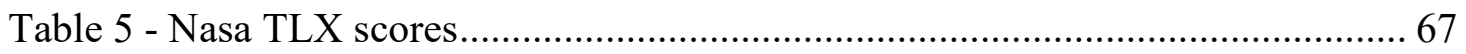




\section{List of Figures}

Figure 1- Nausea Likert scale questionnaire. Participants used this to rank their current nausea level every 1.2 minutes in the preliminary study, and every 2 minutes in the evaluation of viewpoint snapping 25

Figure 2- The software setup environment - The FPS camera is in the center to give users a good range of rotation for visual search, the player only experiences circular movement not linear. 26

Figure 3 Average nausea rating based on different speeds. Error bars show $\pm 1 S E \ldots . .29$

Figure 4 Viewpoint snapping. a) current position of the camera b) camera position, after $22.5^{\circ}$ snap to the next viewpoint. The closed eyes image indicate the fading transition, during which, the screen darkens 32

Figure 5 - Customized Software environment for preliminary study. The green circle is the starting point and the red circle is endpoint. The small yellow markers are the flags. 35

Figure 6 - Camera view - The user should reach to the active flags when the experiment starts. Only 1 flag is activated and when the participant reached that flag, the next one will be activated after that. Score counter and timer are active to show the status of the game to the users. 35

Figure 7 Hardware setup, depicting a participant taking part in the experiment. Participants were seated on a fixed chair to avoid any movement or real body rotation. 42 
Figure 8 The top row of images shows a standard (non-snapping) viewpoint rotation. The second row shows the same rotation with our viewpoint snapping technique enabled. A fast transition eliminates the intermediate frames: meaning the rotation becomes discrete,

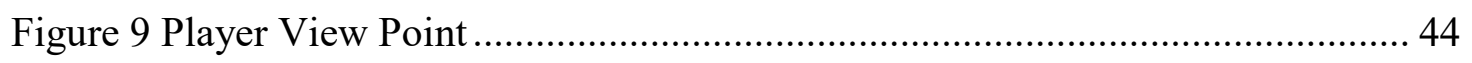

Figure 10 - Nausea Likert scale questionnaire ………………………...................... 45

Figure 11 Box plot of total SSQ Scores. Lower Score is better.................................. 47

Figure 12 Total nausea differences as a function of time. Error bars show standard error. 48

Figure 13 Error rate by viewpoint snapping. Error bars show $\pm 1 S E \ldots \ldots \ldots \ldots \ldots \ldots \ldots \ldots \ldots . . . .49$

Figure 14 - Oculus touch controller and its controller mapping. ................................. 55

Figure 15 Hardware setup, depicting a participant taking part in the experiment. The chair was locked to avoid real body rotation. 56

Figure 16 - Overhead view of virtual environment and waypoints - The green cones served as single waypoints and the blue cones served as double waypoints (i.e., the participant must travel to 2 blue cones in these trials) 57

Figure 17 - Cursor as an indicator for users' teleport point 59

Figure 18 - (Left) Single waypoint trial: The participant starts at the red circle (start position) and travels to the green circle. $\mathrm{R}$ is the ray cast line and $\mathrm{P}$ is the point selected by participants. The distance between P and the start position is PE (Pointing Error). (Right) 
Double waypoint trial: The participant starts at the red circle and moves to the first blue waypoint, then the second one, in sequence. Participants performed the point selection task after reaching the second waypoint. PE is calculated in the same fashion as single waypoint trials 60

Figure 19 - Nausea (discomfort) Likert scale on-screen questionnaire. 62

Figure 20 -Box plot of total SSQ Scores. Lower Score is better. 63

Figure 21 Average nausea scores as a function of exposure time. Error bars show standard error. 64

Figure 22 Average Pointing Error (PE) by translation snapping. Error bars show $\pm 1 S E$.

Figure 23 Total SSQ score between 2 groups TS-NTS and NTS-TS. 66 


\section{List of Appendices}

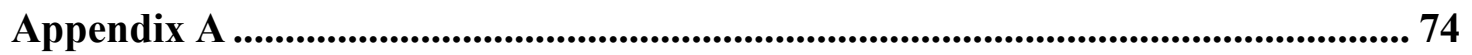

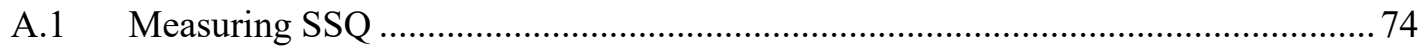

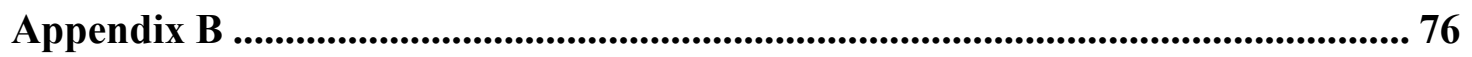

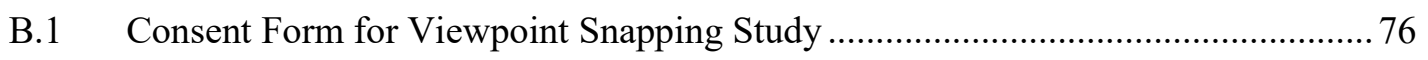

B.2 Consent form for Translation Snapping technique............................................. 78

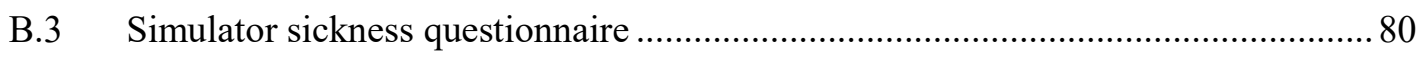

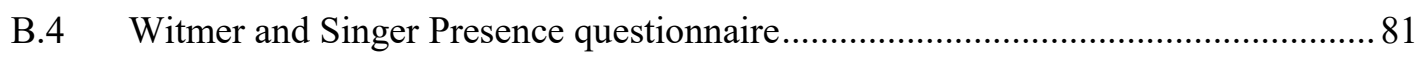

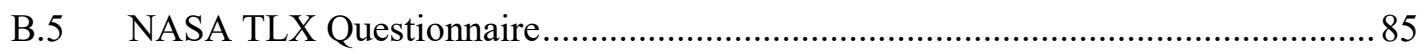

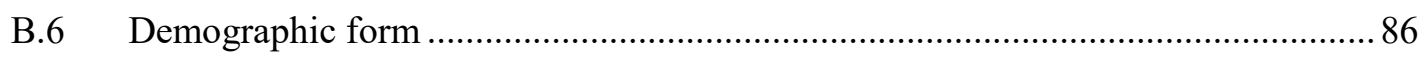




\section{Chapter: Introduction}

Due to advent of low-cost head-mounted displays (HMDs) and tracking solutions, virtual reality (VR) is now accessible to more people than ever. VR has long been used in many application areas such as healthcare, entertainment and scientific visualization [5, 37]. More recently, game companies are developing VR versions of console and PC games, such as Serious Sam VR (developed by Croteam ${ }^{1}$ ) or Resident Evil 7 (developed by $\mathrm{CAPCOM}^{2}$ ). VR offers unique benefits over other 3D (e.g., desktop) systems. Most notable is the immersive qualities of VR, which induces a sense of presence - the psychological phenomenon of feeling as though you are in the virtual place [51].

Presence is important in many VR applications, such as phobia treatment [51] and training [5]. Its importance to gaming is particularly timely, due to the recent excitement around VR gaming. Similarly, the longstanding problem of cybersickness is an increasingly important issue $[12,37]$ due, in part, to the potentially long VR exposures gamers may be willing to subject themselves to experience this new form of gaming. Moreover, joystickbased virtual movement (e.g., via the Oculus Touch joystick) where the user is stationary is commonly used in games. Yet, this mismatches virtual and physical motion; as will be discussed in depth in Chapter 2 - section 2.3, such mismatches yield notably worse cybersickness than walking systems (e.g., the HTC Vive). Cybersickness presents a variety

\footnotetext{
${ }^{1} \mathrm{http}: / /$ www.croteam.com/

${ }^{2}$ http://www.capcom.com/
} 
of symptoms such as nausea, eye strain, headache, sweating, disorientation, or even vomiting [37].

Due to improvements in hardware, several technical factors that contribute to cybersickness are reduced, such as latency and jitter $[12,37]$. In addition, HMDs that support large-scale tracking areas, such as the HTC Vive, allow users to walk naturally, further reducing cybersickness. However, stationary VR setups (e.g., Oculus Rift) are still common, as they offer several benefits over walking systems. These include lower cost (especially for hardware setup), smaller space requirements for tracking, and they are more appropriate for tasks that need longer exposure time. Cybersickness limits training effectiveness in simulators and may have negative effects on performance and learning. For example, in flight simulators may minimize head movement which causes poor transfer of training to real situations [22]. Needless to say, cybersickness is an ongoing and important problem in the use of stationary VR systems. The objective of this thesis is to evaluate the effectiveness of techniques based on discrete viewpoint movement, intended to reduce the onset of cybersickness when navigating virtual environments in such scenarios.

VR game developers have used several techniques $[15,19,63]$ to combat cybersickness in stationary VR setups. In this thesis, we assess the effectiveness of a new class of techniques that has recently been used in some VR games to reduce cybersickness. We refer to our techniques as viewpoint snapping and translation snapping, although other researchers [50] have called similar approaches discrete viewpoint control (a more general term we also employ). Developers have proposed similar approaches such as comfort mode 
used in Serious Sam VR or the discontinuous rotation used in Resident Evil 7. There is anecdotal evidence of the effectiveness of these techniques; we study this formally for use with both viewpoint rotation and translation. The premise of the technique is based on reducing optical flow and inhibiting vection (illusion of self-motion) by employing discrete movement [53] to reduce cybersickness. However, despite the expected benefits of this technique, we also hypothesize that it may negatively influence user performance and presence levels. In respect to this, we measure user preferences and performance with two different user studies. In addition, it is unclear how effective (if at all) viewpoint snapping and discrete movement is at reducing cybersickness. Moreover, there are potential spatial updating implications of employing the technique. After all, the illusion of VR relies on accurately simulating the human perceptual system; discrete movement actively breaks this model.

\subsection{Contributions}

We propose two potential solutions for cybersickness in VR environments using a firstperson view (similar to first-person shooter or FPS $^{3}$ games) for rotational and translational movements. Both techniques work based on discrete movement, rather than continuous movement. We call the discrete rotational movement technique viewpoint snapping and the discrete translational movement technique translation snapping.

\footnotetext{
${ }^{3}$ First Person Shooting
} 
Viewpoint snapping operates when the viewpoint is turning due to moving a mouse or other input device (e.g., joystick) that does not yield correct vestibular cues. Viewpoint motion from head-tracking is excluded since it does yield consistent visual and vestibular information. Translation snapping is implemented in a similar fashion, using discrete movement (short jumps) for translational displacement, during both forward and backward gaze directed movement. With both techniques, the basic idea is to artificially reduce vection (illusion of self-motion) by quickly transitioning the viewpoint, reducing continuous viewpoint motion by skipping frames.

These techniques are easy to implement at low cost and are thus potentially attractive for developers. The techniques can be applied in setups with limited tracking space prohibiting natural movement, and potentially even for users incapable of walking [19]. Since our proposed techniques reduce vection, users can experience lower levels of cybersickness and hence have longer exposure times. This can help them acclimatize to VR through repeated exposure. The techniques could also be a good secondary interaction method for use in comfort mode in many VR applications and games.

Both techniques were designed based on the results of preliminary experiments to assess operational parameters (e.g., snapping range, speed, etc.) of the techniques. In addition, we ran two user studies to evaluate the effectiveness of each technique at reducing cybersickness. Our results show that overall, these techniques do reduce cybersickness with limited impact on user preference and performance. 


\subsection{Outline of Thesis}

This thesis is divided into 6 chapters. Chapter 2 presents a comprehensive literature review on cybersickness in virtual reality, including causes, factors, and theories behind it, and related phenomenon such as simulator sickness. We also discuss potential solutions proposed in prior research and compare these approaches to our techniques.

In chapter 3, we describe the implementation details of our two cybersickness reduction techniques, viewpoint snapping, and translation snapping. This chapter also includes two preliminary experiments that inform the design of our techniques. These experiments establish thresholds within which to operate both techniques as well as functional parameters of the techniques (i.e., the range of snapping).

Chapters 4 and 5 present experiments evaluating the effectiveness of both techniques. As mentioned earlier, these user studies were intended to not only assess the effectiveness of the techniques at reducing cybersickness, but also user preference and task performance with them. Both chapters include participants' information, procedure, apparatus, design, results, and discussions.

The final chapter summarizes findings and proposes design recommendations for discrete movement and potential benefits of this technique in VR to reduce cybersickness. We also discuss limitations of our research and provide suggestions for future studies. 


\subsection{Associated publications}

1. Farmani Yasin, and Robert J. Teather. "Viewpoint Snapping to Reduce Cybersickness in Virtual Reality.” Proceedings of Graphics Interface 2018, pp.159 - 166 , ACM, Canadian Human-Computer Communications Society, 2018. [17]

2. Farmani, Yasin, and Robert J. Teather. "Player performance with different input devices in virtual reality first-person shooter games." In Proceedings of the 5th Symposium on Spatial User Interaction, pp. 165, ACM, 2017. [18] 


\section{Chapter: Related work}

\subsection{Cybersickness}

Cybersickness is a common side effect of video games, virtual reality systems and driving simulators [35]. Cybersickness presents a variety of symptoms, such as nausea, headache, pallor, sweating, dry mouth, heavy-headedness, disorientation, vertigo, ataxia, and in extreme cases, vomiting $[37,56]$.

Motion sickness, simulator sickness, and cybersickness produce similar symptoms, although the cause of each is different. Motion sickness commonly occurs when traveling by car, riding amusement park rides, or even sitting on a spinning chair [12]. Simulator sickness, as implied by its name, mostly occurs in flight and driving simulators. McCauley and Sharkey [41] coined the term cybersickness, describing it as motion sickness that occurs in virtual environments (VEs). Stanney et al. [57] report that the severity of cybersickness symptoms is three times greater than simulator sickness, but also that the profile of symptoms is different. For instance, disorientation is more predominant in cybersickness, while oculomotor symptoms are less pronounced [58]. According to Stanney and Kennedy [58], many VE users reported total sickness (TS, the sum of scores for the SSQ - see Appendix A.1) in comparison with simulator users. They stated that the average TS score for VEs is between 20 and 50 while this the average TS score for simulators is around 10 [56]. 
Vision is a major contributing factor in cybersickness. Unlike classical motion sickness, cybersickness can occur strictly with visual stimulation and no vestibular stimulation [47]. Because of this, some researchers $[34,35]$ have referred to it as visually induced motion sickness (VIMS). According to Keshavarz et al. [34], the symptoms associated with vection are also called visually induced motion sickness (VIMS). VIMS is similar to traditional motion sickness but usually occurs in the absence of physical movement. So, at this point, we need to mention the definition of vection and its relationship with cybersickness.

\subsection{Vection (Illusions of Self Motion)}

Cybersickness occurs when the user visually perceives that they are moving through a virtual environment, despite the fact that they are physically stationary. This illusion of self-motion is also called vection [22, 55], which Tschermak [61] defines as a "powerful illusion of self-motion induced by viewing optical flow patterns". Vection can be experienced while watching a moving train and "creates the illusion that one's own stationary train is moving" [34].

Traditionally VIMS has been called vection induced motion sickness, because based on sensory conflicts theory (see section 2.3.1) [22,55], situations involving conflicting visual, vestibular, and/or proprioceptive perceptual information create cybersickness and related

phenomenon such as simulator sickness [33]. In such situations, like in VR, the visual system perceives the illusion of self-motion, while the vestibular system indicates that the body is stationary with respect to gravity and position [22].

There are two types of vection that are common in VR systems: circular vection and linear vection. Circular vection occurs during camera rotation $[22,60]$ when the scene is 
moving around the viewpoint. Linear vection occurs during movement in a linear direction.

For example, this occurs when the viewer is moving forward or sideways.

\subsubsection{The Relationship Between Vection and Cybersickness}

Previous studies have shown that changing vection (speed and direction) can induce more severe sickness than steady, consistent, vection caused by walking or turning at a constant speed or in the same direction [3]. Noting this, Dorado and Figueroa report that climbing a ramp in VR will yield lower levels of cybersickness than climbing stairs [15] because it reduces vection. According to Davis et al. [13], comparing two different scenarios with an Oculus Rift DK1 and DK2 indicated that more complex and more realistic sceneries also yield higher levels of cybersickness. Visual flow increases with the function of velocity and scene details and the reduction of height above terrain. According to Johnson [27], when a flight simulator is passing near the ground, there is a greater chance of visual flow and vection to occur.

A study by Kemeny et al. [29] revealed that rotational movement causes greater sickness levels compared to translation movements in driving simulators. These results are echoed by Trutoiu et al. [60], who indicate that circular movements/rotations cause maximum cybersickness compared with translation movements in different directions. Speed, however, is also a critical factor.

\subsubsection{The relationship between vection speed and cybersickness}

Previous studies $[23,30]$ have shown that vection and associated symptoms such as cybersickness are significantly affected by movement speed and do not necessarily 
continuously increase with rotation speed. For example, Hu et al. [24] conducted a study using an optokinetic drum with black and white stripes, where they varied circular vection speed from $15 \%$ s to $90 \%$. Their results indicate that as rotational speed increased, symptoms of induced vection, including simulator sickness, increased, peaked, and then declined, with peak symptoms occurring at a rotation speed of $60 \%$. This result indicates that vection and simulator sickness increase to a point, then stabilize. They also suggest that at a rotational speed of $200 \% / \mathrm{s}$, the viewer no longer experiences vection. In addition, So et al. [55] report that the time for vection to occur is reduced for speed changes from $3 \mathrm{~m} / \mathrm{s}$ to $10 \mathrm{~m} / \mathrm{s}$. After $10 \mathrm{~m} / \mathrm{s}$ it stabilizes.

\subsection{Cybersickness Theories}

A detailed discussion of the vestibular system, which is responsible for human balance and is located in the inner ear, is provided by LaViola [37]. For the purpose of this thesis, we instead focus on the main theories as to the causes of cybersickness. Explaining the functionality of the vestibular system is outside the scope of this thesis.

\subsubsection{Sensory Conflicts}

The "sensory conflict" theory is the oldest and most widely accepted theory as to the cause of cybersickness and it was introduced by Reason and Brand [6]. According to this theory, information that is provided by the vestibular system conflicts with that provided by the vision system. For instance, consider a user of a driving simulator. The optical flow patterns of the road, buildings, and other parts of the virtual environment move past the users' periphery giving them a sense of vection (illusion of self-motion). In this scenario, 
the visual system relays information to the user indicating that they are moving in a specific direction. They will accelerate when pushing the gas pedal or decelerate when using the simulator's breaks; however, the vestibular system cannot provide any information indicating any movement. As a result, the user does not get the intended response, which may yield simulator sickness [37].

There are two main problems with the sensory conflicts theory. First, although the theory states that conflicts between vestibular and vision system cause cybersickness, it cannot answer why some individuals feel sick, while others do not. In addition, it cannot predict situations that will cause cybersickness, nor its severity. Finally, there is no explanation as to why these conflicts make someone sick [37].

\subsubsection{The Poison Theory}

The 'poison theory', introduced by Triesman [59], provides an evolutionary explanation as to the cause of cybersickness. According to this theory, ingesting poison can cause physiological responses that involve vestibular, vision, and other sensory inputs. This physiological response is to remove the contents of the stomach, in an effort to eliminate the poison. This could be the main reason that nausea is the most common symptom of cybersickness.

Like the conflicts theory, this theory does not explain why some users feel sick and others do not. In addition, it does not explain "why people who get sick in virtual environments do not always have an emetic response" [37]. 


\subsubsection{The Postural Instability Theory}

Riccio and Stoffregen [49] proposed the postural instability theory, and primarily has focused on the postural stability of humans in an environment. According to this theory, uncontrolled "movement of perception and action system are minimized" [37, 49]. A good example is walking on ice vs. walking on concrete. The mechanism of walking on ice is quite different from concrete; if a person tries to walk on ice exactly like they do on concrete, they will likely slip and fall down. Consequently, people must change their walking pattern on ice in order to control their postural stability. Similarly, changing environments from the real world to virtual reality could also yield postural instability, which may be another cause of cybersickness.

The postural instability tries to refute sensory conflicts theory. According to Riccio and Stoffregen [49], the main reason cybersickness occurs is not sensory conflicts. They argue that if there are no sensory conflicts between the visual and vestibular systems, they should get redundant information. Alternately, if there are sensory conflicts, they should get nonredundant information. However, the theory does not provide any explanation as to why some people with non-redundant information do feel sick [37, 49]. Thus, more research needs to be conducted to check the validity of postural instability.

\subsection{Contributing Factors to Cybersickness in VR}

There are many factors that have an impact on cybersickness, but one of the most comprehensive categories was introduced by Kolasinski et al. [16] in the context of driving simulators. They suggest that the factors that contribute to the potential severity of sickness can be categorized into three groups: 
1) The simulator and technology problems, such as display or image refresh rate, and field of view.

2) Simulation task, which refers to the degree of control, the ability to turn, and the scenarios.

3) Individual factors, such as gender, motion sickness background, and experience.

A summary of similar factors can be seen in Table 1, as presented by David et al. [12]. We will next discuss each category of factors.

\begin{tabular}{ccc}
\hline Individual Factors & Task Factors & Device Factors \\
\hline Age & Lag & Control \\
Gender & Flicker & Duration \\
Illness & Calibration & \\
Posture & Ergonomics & \\
\hline
\end{tabular}

Table 1- Factor categories contributing to cybersickness [12]

\subsubsection{Technology and simulator problems}

Device factors like screen flicker, latency, and position tracking were the most notable technology issues in both VR and simulators systems. New generation HMDs include various technological improvements (such as reduced latency) that have reduced these problems [37]. Display flicker, due to low refresh rates, was one of the factors that caused eye fatigue in older VR systems. Recent displays with faster refresh rates have decreased this problem [16]. Oculus's best practice guide document [45] suggests that low latency, high frame rates, and short-persistence display can drastically decrease cybersickness. 
Field of view (FOV) is another important factor that impacts both the presence and severity of cybersickness. This is usually related to HMDs, and a large FOV can yield greater sickness than smaller displays [46]. Seay et al. [52] studied simulator sickness in the presence of multiscreen driving simulators, and found that cybersickness is correlated with the field of view. In other words, although a larger FOV can increase user presence, it can cause simulator sickness (SS) as well ${ }^{4}$. Based on this observation, Fernandes, and Feiner [19] proposing a cybersickness reduction technique relying on a dynamically changing FOV. They argue that stationary VR setups are important because they are more inclusive of people with disabilities. They further note that searching a large virtual environment is difficult with real walking, due to fatigue. They found that by reducing the FOV while the user is moving, cybersickness was significantly reduced, which can further increase the level of adaption to VR. It also maintained user awareness without negatively affecting subjective levels of presence in VR [19].

The use of motion platforms is another solution that has the potential to reduce cybersickness. By adding motion platform, the user can experience consistent visual and vestibular simulation; however, motion platforms can sometimes cause motion sickness if motion cues are not aligned properly with presented stimuli [37]. Direct vestibular, rest frame stimulation and adaptation/habituation could also serve as solutions, but each requires more research and have limitations [37]. For an example of adaptation, according

\footnotetext{
${ }^{4}$ They suggested that large field of view is "double-edged sword". Although FOV can increase presence, but it can increase risk of cybersickness due to increasing optical flow in the periphery.
} 
to Stanney and Kennedy [56], when the user returns to the real world, they are still adapted to VR and must adapt back to the real world. As a result, after effects can still co-occur. In addition, it is not pleasant for the user to feel sick in each session when adapting to an environment.

\subsubsection{Simulation Tasks}

Control and duration are two important factors regarding tasks in VR environments. Stanney and Kennedy [56] argue that exposure durations should be minimized and that gradual exposure over longer periods of time help users adapt to VR. They advise that the intervals between sessions should be two to five days for adaptation. Most researchers believe that tasks that need too much rotational movement, or forward and backward movement, and acceleration should be avoided until the user totally adapts to VR [45, 56].

The second important factor is how the user controls their virtual movement and performs tasks. When users have control of their tasks, they can predict future motion and are less susceptible to cybersickness. Those who do not have control of their motion are more prone to cybersickness $[12,16]$. This is similar to real motion sickness, as demonstrated by the fact that passengers are more likely to experience motion sickness than drivers.

\subsubsection{Individual factors}

Gender and age are two individual factors that have been extensively studied by different researchers $[1,43,44]$. Despite this research, no specific patterns indicate a relationship between age and cybersickness [1]. For example, Reason and Brand [6] stated 
that motion sickness susceptibility is greater between the age of 2 to 12 years old, while older people are less susceptible to cybersickness. However, Liu et al. [38] report contradictory results and suggest that the frequency and severity of cybersickness and its symptoms increase linearly with age. In other words, older people are more likely susceptible to cybersickness. In addition, Park et al. [43] found no relationship between age, gender and cybersickness, and no significant difference in SSQ scores. As for gender, since women have a larger field of view, this increases the likelihood of flicker and susceptibility to cybersickness, which can sometimes be exacerbated due to hormones [27, 37].

Studying individual factors has its own difficulties. First, researchers need to recruit a wide range of participants, and individual factors like health or alcohol use can increase susceptibility to cybersickness [12].

\subsection{Measurement of Cybersickness}

In general, two methods are widely used to measure cybersickness: subjective and objective methods. Both are critical to understanding this issue.

\subsubsection{Subjective Measurement of Cybersickness}

The earliest subjective model of motion sickness measurement was the Pensacola Motion Sickness Questionnaire [28]. The Pensacola Questionnaire calculated the sum of induvial ratings of dizziness, headache, warmth, sweating, drowsiness, salivation, and nausea. The Pensacola questionnaire was revised several times, and after major revisions, an alternative 16 item Simulator Sickness Questionnaire (SSQ) $[12,31,56]$ replaced it. 
This questionnaire was validated in 1993 by Kennedy et al. [31], and based on three categories (see Table 2): the oculomotor cluster, which includes eyestrain, difficulty focusing, blurred vision and headache; the disorientation cluster, which includes dizziness and vertigo; and the nausea cluster, which includes stomach awareness, increased salivation and burping [12]. See Table 2 for a summary of symptom clusters.

\begin{tabular}{ccc}
\hline Disorientation & Nausea & Oculomotor \\
\hline Dizziness & Stomach awareness & Eyestrain \\
Vertigo & Increased salivation & Difficulty Focusing \\
& Burping & Blurred vision \\
& & Headache \\
\hline
\end{tabular}

\section{Table 2- Example of Symptoms Clusters}

Based on a simulator sickness score (see Table 3), there are three different categories: nausea related score $(\mathrm{N})$, the oculomotor related score $(\mathrm{O})$, the disorientation related score (D). In addition, total sickness score (TS) can be calculated by adding all three factors showing the overall severity of sickness (see Appendix A.1).
1. General discomfort
9. Difficulty Concentrating
2. Fatigue
10. Fullness of head
3. Headache
11. Blurred vision
4. Eyestrain
12. Dizziness with eye open
5. Difficulty focusing
13. Dizziness with eye closed
6. Salvation
14. Vertigo
7. Sweating
15. Stomach awareness
8. Nausea
16. Burping

Table 3- 16 items ranked on the SSQ 
There are other questionnaires that are similar to the SSQ, such as the nausea profile and VRSQ. We do not provide a thorough analysis of them here, because they are less widely used than the SSQ, and we do not employ them in our own studies. Davis et al.'s systematic research of cybersickness provides a comprehensive review of this subject [12]. It is important to consider the main limitation of subjective measurements, such a "lack of reliability, response biases, or social desirability" [34]; nevertheless, these measures (especially the SSQ) are still the most common way to measure cybersickness.

\subsubsection{Objective Measurement of Cybersickness}

Several researchers have tried to establish objective measurements for cybersickness via physiological parameters, such as heart rate, electrodermal activity, and gastrointestinal activity. One of the most prominent and comprehensive studies was conducted by Kim et al. [36]. In their study, 61 participants performed a navigation task in VR for 9.5 minutes, after which their bodies were measured. The researchers used the SSQ (pre and post-test questionnaire). In addition, 16 physiological responses were captured before, during, and after the navigation task. The results indicate that "the total severity of cybersickness had a significant positive correlation with gastric tachyarrhythmia, eyeblink rate, heart period, and EEG delta wave and a negative correlation with EEG beta wave" [36].

Researchers commonly criticize the accuracy of sensors and similar measurement devices as a means to objectively measure cybersickness [12], as changes in physiological responses may be related to many factors other than cybersickness. However, sensors can still be used as the second approach for measuring cybersickness. Dennison et al., argue 
that using only objective measurement can only be precise up to $78 \%$ compared to subjective measurement [14].

\subsection{Techniques to Reduce Cybersickness}

Based on speed and vection results and the inconsistent locomotion [28], a viewpoint snapping technique has the potential to reduce the occurrence of cybersickness. However, there is limited literature exploring the benefits of viewpoint and translation snapping in this regard, though there are some commercial games use a similar approach. For example, the games Serious Sam VR and Capcom's Resident Evil 7 both include a 'snap rotation' feature. When activated, this option prevents the player from rotating their viewpoint continuously, instead of snapping their rotation to fixed increments. Mark Scharamm of VR-Bits [62] used a similar approach in a travel technique he called 'Cloud Step' [11]. These techniques have not been formally evaluated in any capacity.

Several researchers have proposed different approaches to reduce cybersickness during rotation and translation $[9,15,19,29]$. One approach involves adding a depth of field blur effect during rotation, which simulates the focusing of the eyes at a different depth. This requires blurring the out of focus parts of the scene slightly [7]. In a user study, the authors found that rotation blurring [7] did not significantly decrease overall cybersickness (per SSQ). It did, however, significantly delay onset of cybersickness by 2 minutes.

Another technique is the "headlock" [29], which temporarily disables viewpoint rotation during rotation. Head-lock was found to significantly (by about 30\% Total SSQ) reduce cybersickness during rotation in a CAVE environment, as compared to joystick rotation. However, the authors also report that presence scores were significantly reduced when 
using head-lock. Furthermore, participants reported that head-lock was not intuitive compared to joystick rotation. Beyond these issues, both head-lock and rotation blurring only work during rotation; neither sets of authors proposed a related solution for translations.

Another technique mentioned earlier involves changing the field of view during movement [19]. This so-called "tunneling" technique is similar to the approach used by the VR version of the commercial game Serious Sam. However, tunneling was found to not significantly reduce total SSQ scores [19] between two groups in two different sessions. However, raw discomfort scores taken in real-time during the experiment (similar to the nausea scores in our experiments, see Chapters 3 through 5) reveal a reduction in nausea when using the FOV tunneling technique. However, the authors did not quantify user presence, nor is it clear how FOV reduction can affect user performance.

Dorado and Figueroa [15] proposing using ramps instead of stairs. They argue that using steady movements can reduce vection. As a result, users should feel less pronounced symptoms. Using ramps significantly reduced total SSQ scores [15] by between $10 \%$ to $24 \%$, in two user studies. The main reason for this reduction was vection reduction and lowering vection speed (see section 2.2.2 for more information). This technique minimizes changes in vection (both direction and speed), but the study results also indicate increasing speed will increase cybersickness. Presence and performance were not measured, and it is dependent on task and scenario. In addition, it is not clear if applying steady or constant movements at high speed, if ramps would be as effective as in this study. 
In addition, other researchers proposed methods to reduce cybersickness based on increasing sense of embodiment and reducing vection. Chang et al. [9] proposed using rest frame (e.g., using a cockpit) to reduce cybersickness by "delaying the onset of cybersickness by alleviating users' attention or perception load". Furthermore, using a virtual nose or a user avatar can also reduce cybersickness since it increases the sense of embodiment [21], in accordance with the postural instability theory. Chang et al. [9] used rest frames with a virtual roller coaster, and report total SSQ significantly decreased by around $10 \%$ compared to a condition without rest frames. Like other studies, the authors did not evaluate if rest frame affect presence and performance, nor did they address how comprehensively it could be used in other game scenarios.

One limitation of previous work is that nearly all studies on the techniques were only evaluated in terms of their impact on cybersickness. None of these studies [7, 9, 15, 19, 29] provided any results on user task performance, and only one included presence [28]. It is plausible that several of these techniques may severely limit user performance. For example, reducing the FOV $[19,46]$ cuts out parts of the user's view; in a fast-paced action game, this could prevent the user from seeing an enemy, and thus may impact their ability to react to game events as quickly. Similarly, adding UI elements like cockpits, rest frames, or even a virtual nose occlude parts of the screen. It is unclear how they affect user presence or performance. Finally, some of these techniques (e.g., using a cockpit) are scenariospecific. In other words, they do not generalize to all virtual environments. For instance, a 
vehicle or space ship could be used in a game like Valkyrie ${ }^{5}$, but in a game like Valiant ${ }^{6}$, a kettle hat should be added to the first person view instead. Neither approach would be appropriate in, for example, a VR medical training simulator. So, each of these techniques either has limitations preventing widespread adoption, or there are still questions about their potential impact on other important aspects of VR environment usage (e.g., task performance and presence).

In this thesis, we ran two user studies with different scenarios. We evaluated discrete viewpoint control in more complex scenarios like an FPS game and a path integration task. In addition, we studied the effect of our technique on both user presence, and performance in the prescribed tasks. To our knowledge, no prior study investigated thresholds for discrete rotation and translation. It is as yet unclear angle or jump distance is sufficient for rotation and translation snapping. The Oculus Best Practice Guide [45] proposes a $30^{\circ}$ snapping increment for discrete rotation; but, the reason of proposing this number is not clear, and likely developed informally via ad hoc testing. We ran two preliminary studies to find best thresholds in terms of user performance, preference and comfort. In addition, based on these performance-based studies we ensure that the discrete movements are not disorienting.

\footnotetext{
${ }^{5}$ https://gaming.youtube.com/game/UCs9XYBocLgnrQIuWKf0zuCw

${ }^{6} \mathrm{https}$ ://store.steampowered.com/app/344180/Valiant/
} 


\section{Chapter: Preliminary Studies, and Implementation of Techniques}

\subsection{Theoretical Basis}

Both vection and cybersickness are generated when watching moving scenes. As discused in detail (section 2.2.2) increasing vection speed can increase cybersickness. Conversely, reducing vection can also reduce cybersickness [13, 15, 34]. For example, Hu et al. [25] report that "as the rotating speeds of an optokinetic drum ${ }^{7}$ with black and white stripes changed from $15 \%$ s to $90 \%$, symptoms of vection induced sickness increased".

Discrete movement is one method of reducing or inhibiting vection [53], and is a good candidate for further study in designing techniques to reduce cybersickness. There is anecdotal evidence of the effectiveness of discrete movements from industry (see e.g., Serious Sam, Resident Evil, Clouds Step technique). In addition, there are no prior studies formally evaluating discrete movement for both translation and rotation as a method to reduce cybersickness, nor establishing operational parameters for such techniques.

Inconsistent locomotion [53] has been shown to inhibit vection by reducing optical flow. Techniques such as reducing the FOV operate on a similar principle, reducing optical flow by decreasing the visible imagery [19], rather than skipping imagery entirely. We instead propose to reduce cybersickness with discrete movements. In other words, the number of frames observed by users will decrease. However, as mentioned earlier, there are no "obvious" operational parameters to use for such snapping. For example, what snapping

\footnotetext{
${ }^{7}$ Optokinetic drum is traditionally used for measuring vection while participants rotating.
} 
distance/angle should be used? At what rotation or translation speed should such techniques work? Previous industry attempts (e.g., the Oculus Best Practice Guide [45] mentioned $30^{\circ} / \mathrm{s}$ ) seem to rely on anecdotal and ad hoc testing methods to establish such parameters. We propose to formally study these in designing discrete motion techniques.

In order to design the viewpoint snapping and translation snapping technique, we ran two preliminary user studies. These studies were intended to provide parameters within which to operate the techniques. In the case of viewpoint snapping, this is based on camera rotation speed. In the case of translation snapping, our goal was to determine the optimal jumping distance.

\subsection{Discrete Rotation}

We first describe our discrete rotation technique or viewpoint snapping. This section includes a description of a preliminary experiment designed to determine thresholds to active viewpoint snapping based on camera speed and user comfort. We then describe the viewpoint snapping mechanism in detail.

\subsubsection{Preliminary Experiment}

We present a first study motivating the design of our viewpoint snapping technique. This was not intended to evaluate the effectiveness of the technique, but rather to establish an approximate rotation speed threshold within which to activate our viewpoint snapping. This preliminary study was intended to determine user preferred speed and discomfort levels using a nausea questionnaire similar to that used by Fernandes and Feiner [19], and 


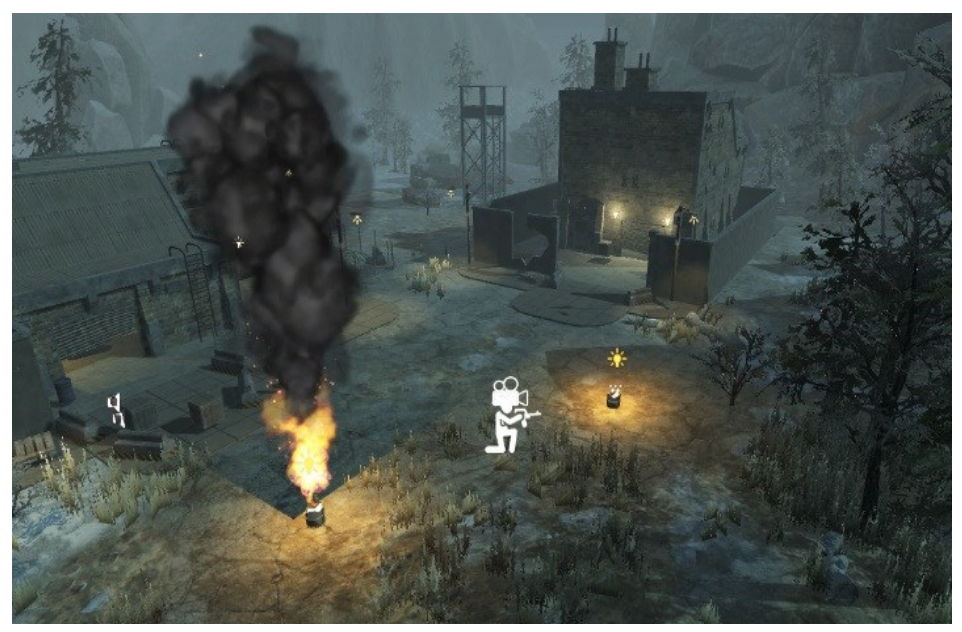

Figure 2- The software setup environment ${ }^{8}$ - The FPS camera is in the center to give users a good range of rotation for visual search, the player only experiences circular movement not linear.

\subsubsection{Procedure}

Participants first signed a consent form and the experimental method was explained to them. Participants were then asked to rate their level of nausea from 1 to 10 at the end of each minute trial using the survey described above. Each trial consisted of 1.2 minutes of exposure to a particular rotation speed. The objective was not to quantify their level of vection, but rather to assess at which rotational speeds they experienced the greatest degree of nausea. As Keshavarz et al. [34] report, it is difficult to quantify vection directly because participants usually have different experiences or may report in a biased or unreliable fashion.

Participants were instructed that they could withdraw and stop the experiment at any time, especially if they experienced extreme symptoms. The participant then puts on the

\footnotetext{
${ }^{8}$ https://www.assetstore.unity3d.com/en/\#!/content/59359
} 
Oculus Rift head-mounted display. The camera started rotating while the participant looked forward; during this rotation, they were instructed to hold their head stationary. Every 1.2 minutes (i.e., at the end of each condition), the nausea rating questionnaire (Figure 1) appeared on the screen. The participants rated their nausea from 1 to 10 using the Oculus Touch controller, in the fashion described above. Selecting a nausea score of " 10 " indicated that they wanted to stop and withdraw from the experiment. During the experiment, participants were also asked if they felt as though they were really rotating or not. Upon completion of the experiment, we asked them which rotational speed they preferred the most.

\subsubsection{Design}

The experiment included a single independent variable, rotation speed with 11 levels: $5 \%$ s, $10^{\circ} / \mathrm{s}, 15^{\circ} / \mathrm{s}, 20^{\circ} / \mathrm{s}, 25^{\circ} / \mathrm{s}, 30^{\circ} / \mathrm{s}, 40^{\circ} / \mathrm{s}, 60^{\circ} / \mathrm{s}, 100^{\circ} / \mathrm{s}, 120^{\circ} / \mathrm{s}$, and $200^{\circ} / \mathrm{s}$. The dependent variable was the average level of nausea, as reported by participants using the Likert-scale survey (Figure 1).

\subsubsection{Results and Discussion}

As expected based on previous work [55], higher rotational speeds yielded higher nausea scores (Figure 3 and Table 4). While expected, this data provides thresholds where cybersickness was worst to help inform the design of the viewpoint snapping technique. Based on these findings and previous work [55], the preferred rotation speed was between $15 \%$ and $35 \%$ s (chosen by 10 participants). At higher speeds, participants felt 
uncomfortable. One-way ANOVA revealed a significant difference in nausea scores by rotational speed $(F 10,121=5.1, p=0.0001)$.

\begin{tabular}{|c|c|c|c|c|c|c|c|c|c|c|c|}
\hline \multirow[b]{2}{*}{ ID/speed } & \multicolumn{11}{|c|}{ Rotational Speed $(\%)$} \\
\hline & 5 & 10 & 15 & 20 & 25 & 35 & 45 & 65 & 100 & 120 & 200 \\
\hline $\mathbf{P 2}$ & 1 & 1 & 1 & 1 & 1 & 1 & 1 & 1 & 1 & 1 & 1 \\
\hline P8 & 1 & 1 & 1 & 1 & 1 & 1 & 1 & 1 & 1 & 1 & 1 \\
\hline P6 & 2 & 2 & 1 & 2 & 1 & 1 & 3 & 3 & 4 & 4 & 6 \\
\hline P12 & 1 & 1 & 2 & 2 & 2 & 2 & 2 & 3 & 3 & 3 & 8 \\
\hline P11 & 1 & 1 & 1 & 3 & 4 & 5 & 5 & 3 & 5 & 7 & 8 \\
\hline P1 & 3 & 3 & 4 & 4 & 4 & 4 & 5 & 5 & 5 & 5 & 5 \\
\hline P5 & 4 & 5 & 3 & 2 & 2 & 3 & 6 & 7 & 7 & 8 & 8 \\
\hline P3 & 2 & 5 & 6 & 6 & 5 & 6 & 6 & 7 & 8 & 9 & \\
\hline P7 & 1 & 2 & 5 & 6 & 7 & 8 & 7 & 8 & 9 & 9 & \\
\hline P4 & 1 & 4 & 5 & 6 & 6 & 6 & 8 & 8 & & & \\
\hline P9 & 1 & 3 & 4 & 5 & 6 & 7 & 8 & 8 & & & \\
\hline P10 & 1 & 1 & 8 & 6 & 7 & 8 & 8 & 9 & & & \\
\hline
\end{tabular}

Table 4 - Experiment one - nausea scores by rotational speed. The red square indicates users withdraw from the study. It usually happens at the speed more than $65.3 \%$ s. Three users withdraw at speed of $100 \%$ and two at speed of $200 \%$ s. These are depicted as red cells in the table.

Nine participants reported that at the highest speed $\left(200^{\circ} / \mathrm{s}\right)$, scene details were no longer visible, and the feeling of movement was reduced. Four participants mentioned that they just felt dizzy. One participant mentioned that she "felt rotation from $10^{\circ} / \mathrm{s}$ to $60^{\circ} / \mathrm{s}$, but after that the rotation speed becomes unnatural, details are less obvious, and he just felt more nervous tension or mental strain". Another participant mentioned that he felt more tension from speeds between $40 \%$ s to $100 \%$ s. At the speed of $200 \%$, though, he did not feel any movement at all. As seen in Table 4, at high rotation speeds, several participants withdrew from the study. Three withdrew at $100^{\circ} / \mathrm{s}$, and two more withdrew at $200 \% \mathrm{~s}$. 
Based on these results, a threshold of $25 \%$ sas deemed appropriate to activate viewpoint snapping (Table 4). This threshold was the average of most participants' preferred speeds, which fell between $15 \%$ s and $35^{\circ} / \mathrm{s}$. It also corresponded to the rotational speed where nausea scores started becoming more notable: a score of "4". This was followed by a steady increase in nausea scores (Figure 3).

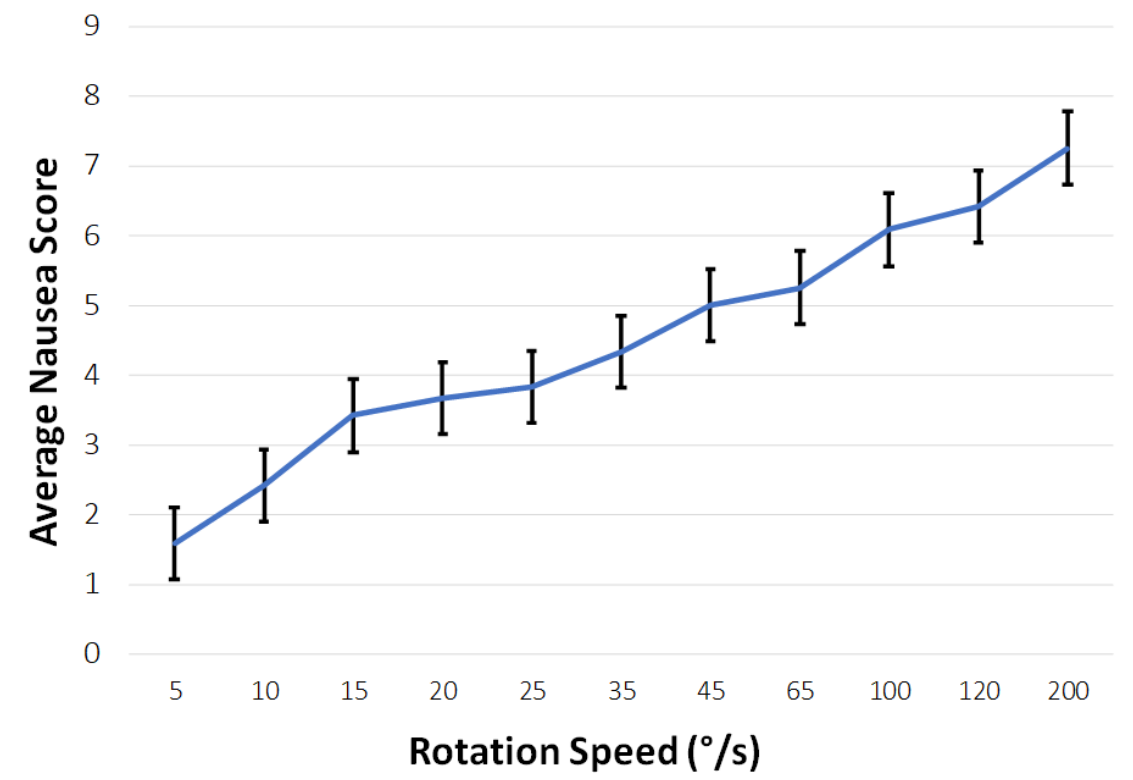

Figure 3 Average nausea rating based on different speeds. Error bars show $\pm 1 S E$.

We note here a possible confound in this experiment, due to the fact that rotational speeds were always presented in the same order, and hence increased with exposure time. We argue that this likely did not influence our results, and that the nausea scores reported were likely due to rotational speed. Exposure time was quite short (less than 15 minutes in total), and likely not long enough to yield cybersickness due to exposure alone [55]. Previous cybersickness researchers noted that nausea scores only significantly increase due to exposure after lengthy periods of time. While the exact duration varies depending on the 
source, the shortest exposure time reported causing cybersickness is 20 minutes [32, 48] and as high as 60 minutes [42]. Given that our total exposure was about 13 minutes (1.2 minutes per condition), exposure time alone was likely not long enough to influence nausea levels much.

Nevertheless, these nausea scores are likely best thought of as approximate due to the potential limitation. However, any difference caused by counterbalancing the order of rotational speeds would be quite small due to the relatively short exposure time. Moreover, counterbalancing the order could potentially expose participants to high rotation speeds immediately upon starting the study, yielding cybersickness right at the onset. Overall, this also suggests that a threshold of $25 \% \mathrm{~s}$ is likely slightly conservative. In other words, viewpoint snapping may be activated at a slightly lower speed than necessary, which is unlikely to influence cybersickness but may slightly affect performance and presence. Given the range of the nausea scores (Figure 3 and Table 4), the best answer is likely to "calibrate" a viewpoint snapping threshold on a per-user basis. This was not practical for the current study but will be investigated in future.

\subsubsection{Viewpoint Snapping technique}

In this section, we describe our viewpoint snapping technique itself. For our experiment (Chapter 4), we used a mouse as an input device to control viewpoint rotation. Input devices (like the mouse or joysticks) induce cybersickness due to visual-vestibular conflicts and vection [34]. As noted by other authors, and described in depth in Chapter 2, cybersickness is strongest in the absence of actual physical movements [12]. The current study employed mouse rather than a joystick since it is more familiar to participants, and allows higher- 
speed position-control rotations, rather than the velocity-control rotations supported by joysticks. However, the technique is expected to work well with either input device. For example, Cloudhead Games ${ }^{9}$ implemented a similar technique using a joystick, while Okleyros ${ }^{10}$ used a mouse. Other similar techniques have been proposed by Oculus, who refer to it as "blinks" or "snap turns"11.

For this initial study, viewpoint snapping was only utilized on a vertical-axis rotation, specifically yaw. Thus, snapping only occurred when the user was turning right or left with rotation speed over the threshold $(25 \%)$, as determined in the preliminary experiment (Section 3.1.1). To activate viewpoint snapping, the current study used mouse movement speed, which corresponds directly to camera rotation speed, to modify the movement direction/gaze direction. When rotating above $25^{\circ} / \mathrm{s}$, the continuous rotation was replaced with a fast fading transition animation between $22.5^{\circ}$ increments. The fading transition was intended to help prevent loss of spatial context by preventing immediate jumps between viewpoint thresholds.

As depicted in Figure 4, this effect behaves as though the user closed their eyes, quickly turned their head $22.5^{\circ}$, and then opened their eyes. In summary, for this technique, no snapping occurs if rotation speed is less than $25 \%$ s. However, if the rotation speed is above $25^{\circ} / \mathrm{s}$ in a given yaw direction, the fading animation starts and the camera snaps in $22.5^{\circ}$

\footnotetext{
${ }^{9}$ http://cloudheadgames.com/

${ }^{10}$ http://doc-ok.org/?p=872

${ }^{11}$ https://developer.oculus.com/design/latest/concepts/bp-locomotion/
} 
increments in the specified direction. Upon reaching the next rotation increment, the fading transition stops: the transition speed at this point is $800 \mathrm{~ms}$.

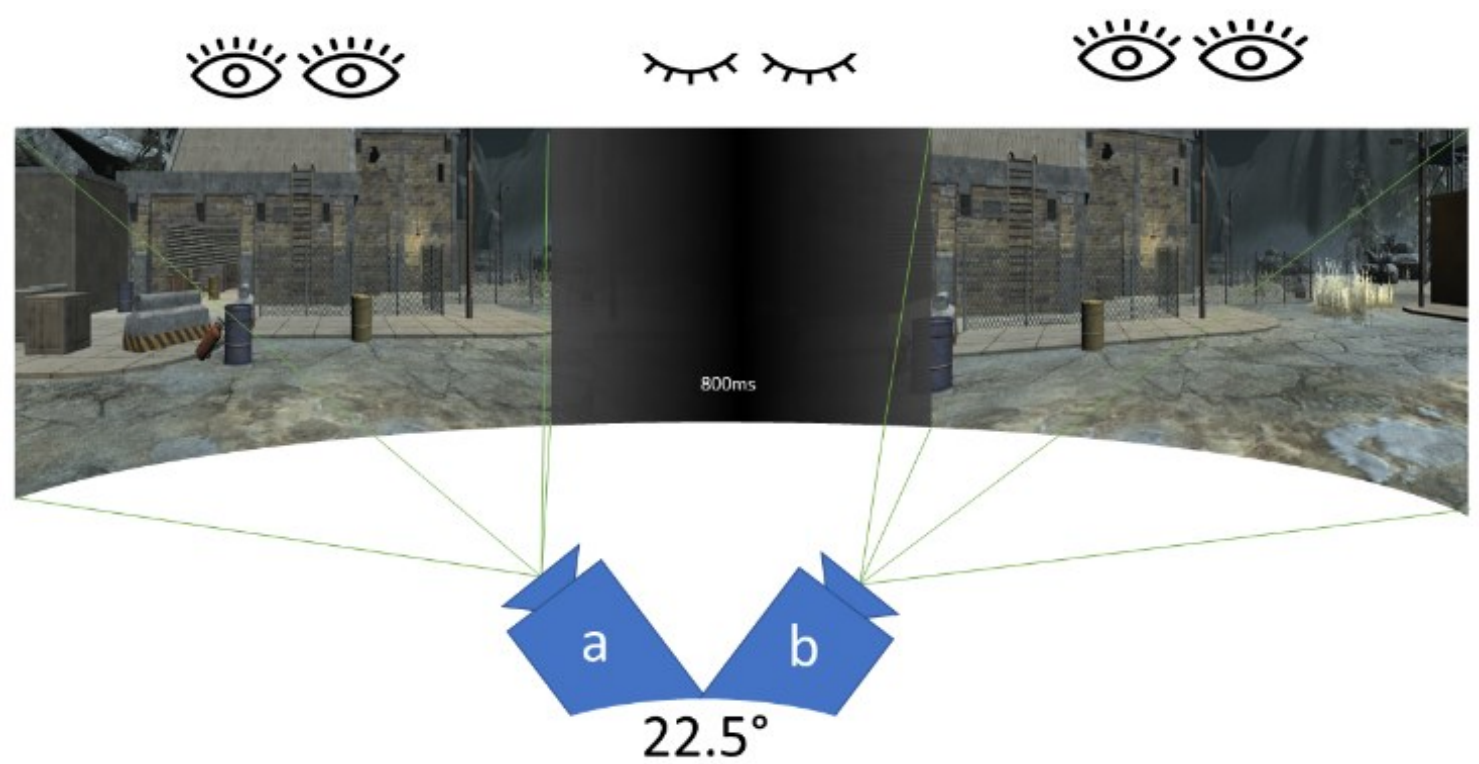

Figure 4 Viewpoint snapping. a) current position of the camera b) camera position, after $22.5^{\circ}$ snap to the next viewpoint. The closed eyes image indicate the fading transition, during which, the screen darkens.

We decided on a $22.5^{\circ}$ increment for snapping based on informal pilot testing. We initially tried a snapping distance of $45^{\circ}$, but found it disorienting [45]. The Oculus Best Practice guide [45] recommends a threshold of around $30^{\circ}$ to prevent user disorientation when using their snap turns. We tested the $30^{\circ}$ range as well, but ultimately changed to $22.5^{\circ}$ as we found it most comfortable. Snapping range is likely dependent on rotation speed and is a topic for future study. 


\subsection{Discrete Translation}

In this section, we present a preliminary study designed to find an appropriate jump distance based on user's preferences and performance. We then explain the translation snapping technique in detail.

\subsubsection{Preliminary study}

To design the discrete translation technique, we ran a preliminary experiment with 9 participants. In this experiment, we evaluated four different jump distances and gathered objective and subjective data. We collected and analyzed the optimal distance traveled, completion time, and participant preference. We used the results of this experiment to inform the design of our translation snapping technique, in particular, the delay between jumps when using continuous movement, and the most comfortable jump distance.

\subsubsection{Participants}

9 participants -4 females and 5 males - between the ages of 18 to $37($ mean $=27.8)-$ were recruited. Each participant was a student and the majority (6 participants) had experienced VR systems between 1 to 10 times. A single participant never used VR before and 2 participants used VR frequently.

\subsubsection{Apparatus}

The experiment was conducted on an ASUS (Model: GL502VS) gaming laptop (i76700 HQ 2.6GHz CPU 2.59 GHz, GeForce GTX 1070 GPU, 32GB RAM) with an Oculus Rift CV1 head-mounted display with 2 tracking cameras. The virtual environment was created and rendered with Unity game engine (version: 2017.1.1f1 Personal). We used an 
Eastern Times Tech T7 gaming mouse as the input device. In order to simulate discrete jumps, participants were instructed to click the left mouse button for a jump forward and right click for jump backward. Upon pressing the button, the participant's viewpoint was instantly translated in the specified direction along the view vector, moving a distance specified by the current jump distance condition (i.e., $0.25,0.5,1.0$, or 2.0 meters).

The current study used the same FPS level demo as in the previous study (see Figure 2) as a base, with experiment-specific customizations. We removed some 3D objects and scenes details to have wider space for navigation as well as to avoid user collisions with objects in the environment. We put 20 flags in the virtual environment denoting a path; participants were tasked with collecting these flags in order (see Figure 5). The flags were initially invisible, and only one flag was active (visible) at a time. When approaching $1 \mathrm{~m}$ of the active flag, it would disappear, and the next flag in the sequence would become active. In this fashion, the participant would follow the path seen in Figure 6, one flag at a time. Upon reaching the active flag, a score counter incremented, and a chime emitted to inform participants that they had reached their goal. The user's point of view is seen in Figure 6 . 


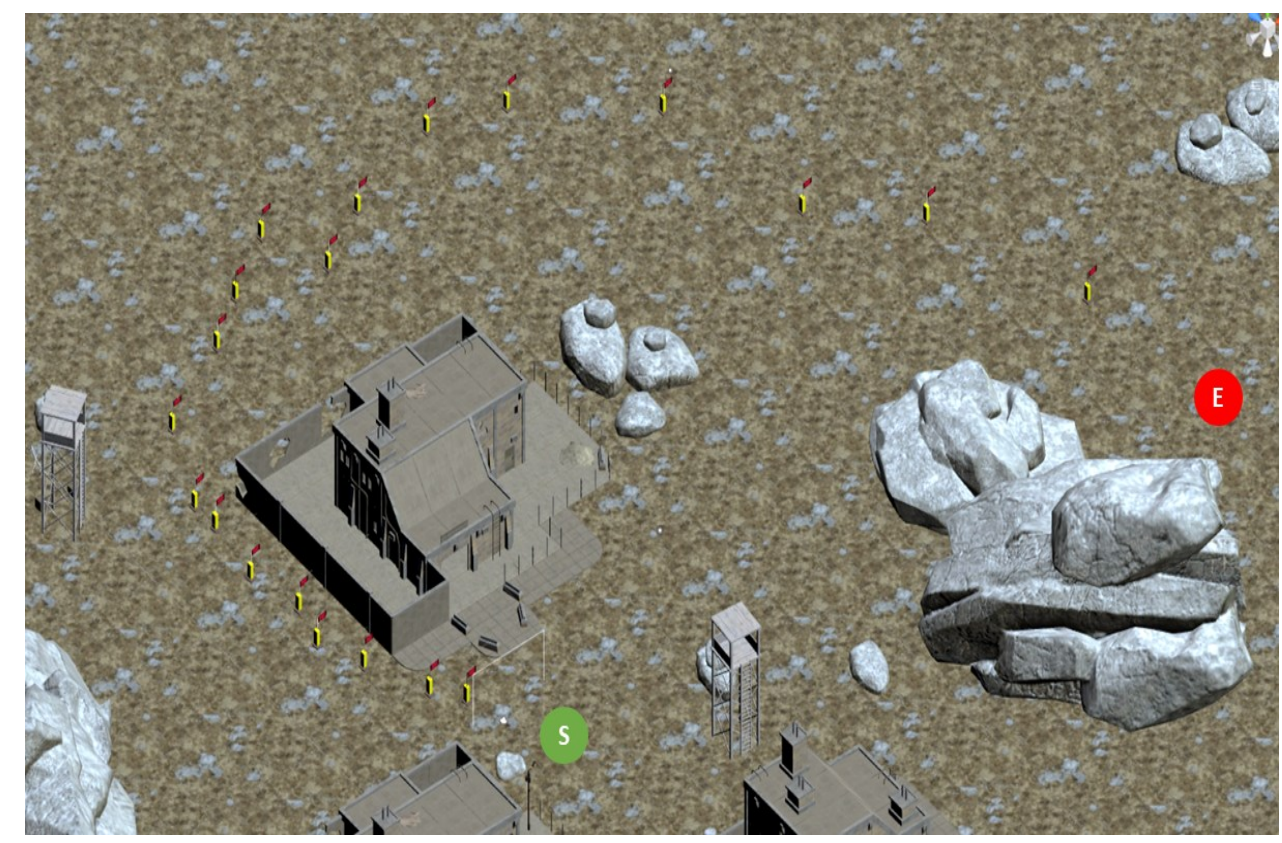

Figure 5 - Customized Software environment for preliminary study. The green circle is the starting point and the red circle is endpoint. The small yellow markers are the flags.

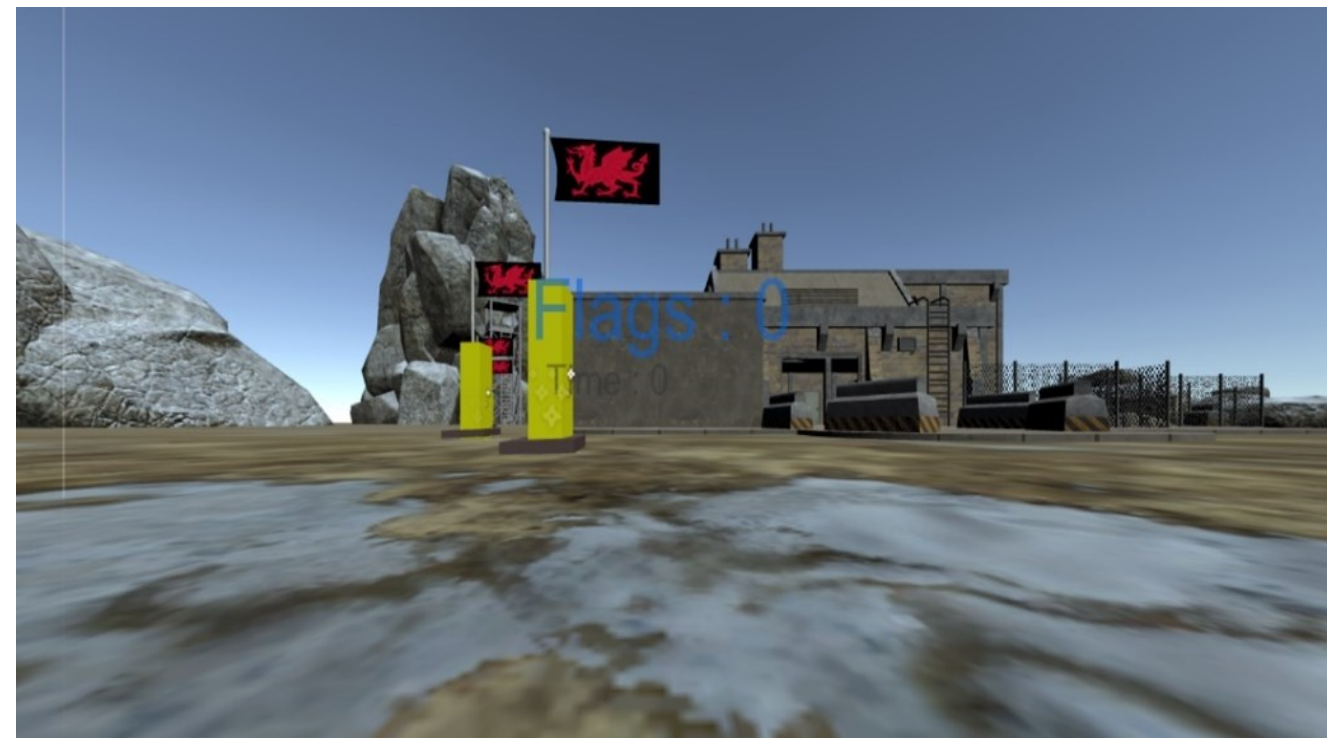

Figure 6 - Camera view - The user should reach to the active flags when the experiment starts.

Only 1 flag is activated and when the participant reached that flag, the next one will be activated after that. Score counter and timer are active to show the status of the game to the users. 
Before the experiment, we calculated the optimal distance from start to endpoint (see Figure 5) using the Unity AI (artificial intelligence) library. Specifically, we used a NavmeshAgent ${ }^{12}$ employing the $\mathrm{A}^{*}$ search algorithm to find the shortest path to the goal.

We considered four different jump distances $(0.25 \mathrm{~m}, 0.5 \mathrm{~m}, 1 \mathrm{~m}, 2 \mathrm{~m})$ to determine which would be more efficient in terms of completion time and traveled distance. Since it is obvious that long jump distances would allow faster travel (and hence shorter completion times), we normalized time with this formula:

$$
\text { Time Score }=\text { Completion time }- \text { Minimum calculated time in each jump group }{ }^{13}
$$

where Completion time is the actual time taken for participants to finish the entire travel task (i.e., collect all 20 flags in order). The minimum calculated time is the lowest time among participants for each jump distance group.

In addition, as the optimal distance was constant (193m) for the environment, the distance score was calculated as:

\section{Distance Score $=$ calculated distance - Optimal distance}

where optimal distance was always $193 \mathrm{~m}$ (as determined by the A* search algorithm the most efficient path to complete the travel task) and calculated distance is traveled distance for each user which is equal or greater than optimal distance.

\footnotetext{
12 https://docs.unity3d.com/ScriptReference/AI.NavMeshAgent.html

${ }^{13}$ We also normalize the scores with Standard Score formula: Time Score $=\frac{\text { Calculated Time }-\mu}{\sigma} ; \mu=$ mean and $\sigma$ is standard deviation of each jump distance group. Result was the same for both formula.
} 


\subsubsection{Procedure}

After signing the consent form, we explained the test conditions to the participants. We asked them to use the mouse as an input device to collect active flags in the environment. We first showed them the control mapping using the mouse. The process was straightforward; pressing the left mouse button moved the participant one step forward, while pressing the right mouse button moved them one step backward, using the current jump length. They were encouraged to adjust their clicking speed so as to yield lower levels of discomfort; i.e., not too fast, and not too slow, at their discretion. They were instructed to collect all 20 flags in each of the four jump distance conditions. Prior to starting the experiment, participants put on the Oculus Rift HMD and practiced the study task for two minutes; these practice trials were not recorded.

After collecting 20 flags and finishing the first jump distance condition, we asked them to close their eyes and rest for 30 seconds. Then we began the next condition. In total, the exposure time took approximately 10 to 12 minutes. Upon completion, we asked participants:

- Which speed did they prefer the most?

- Which one was most comfortable?

Overall, the user study took approximately 25 to 30 minutes. Participants were not compensated. 


\subsubsection{Design}

The experiment employed a within-subjects design with a single independent variable, jump distance, with four levels $(0.25 \mathrm{~m}, 0.5 \mathrm{~m}, 1 \mathrm{~m}$ and $2 \mathrm{~m})$. We counterbalanced jump distance ordering using a Latin square. The dependent variables included the distance score, and time score (see 3.3.1.2 for equations).

\subsubsection{Results and Discussion}

A one-way ANOVA was conducted to compare the effect of jump distance for distance score. ANOVA revealed the effect of jump distance on distance score was statically significant $(F 3,32=17.7, p<0.05)$. Post hoc comparisons using the Tukey HSD test indicated that the mean distance score for the $0.25 \mathrm{~m}$ jump distance $(\mu=3.6, S D=0.8)$, $0.5 \mathrm{~m}$ jump distance $(\mu=5.6, S D=0.9)$ and $1 \mathrm{~m}$ jump distances $(\mu=9.5, S D=1.2)$ were all significantly better than the 2 m jump distance $(\mu=23.7, S D=3.9)$, but not significantly different from each other.

In addition, a one-way ANOVA was conducted to compare the effect of jump distance on time score. There was not a significant effect of jump distance on time score $\left(F_{3}, 32=\right.$ 2.5, $p=0.075)$. The mean time scores were: $0.25 \mathrm{~m}(\mu=61.2, S D=11.1), 0.5 \mathrm{~m}(\mu=29.5$, $S D=9.8), 1 \mathrm{~m}(\mu=23.8, S D=6.3)$ and $2 \mathrm{~m}(\mu=37.5, S D=12.8)$.

Finally, we asked participants their preferences as to which jump distance was more comfortable and most preferred. Eight of the nine participants preferred a jump distance of 1 meter, with the $0.25 \mathrm{~m}$ jump distance coming in second. 


\subsubsection{Translation Snapping technique}

Based on the results of the preceding experiment, we decided to use a jump distance of 1 meter for our translation snapping technique. Before implementation, we wanted to ensure that for the main user study, both test conditions used the same input method, which involved holding the mouse button to move forward. Hence, rather than pushing a button for several times, participants can more easily hold a button. This approach makes the translation snapping more comfortable and less fatiguing on the finger. However, we decided to add a timeout delay between jumps based on how quickly participants clicked the mouse in the preliminary experiment. This ensures that the translation snapping did not operate continuously, but rather, would happen at regular intervals while the mouse button was held down.

As indicated earlier, we instructed participants in the preliminary study to click the mouse at a speed that is comfortable (i.e., not too fast or too slow). We used this speed to determine the average number of clicks per second, which in turn yields the average number of jumps ${ }^{14}$ performed each second. On average, with the $1 \mathrm{~m}$ jump distance, participants clicked 2.4 times per second. Phrased differently, they jumped roughly every $416 \mathrm{~ms}$ on average. We thus incorporated this delay into our translation snapping technique; holding down the mouse button would issue a jump event every $416 \mathrm{~ms}$. This ensures we have a discrete movement with analog input.

${ }^{14}$ One jump is equal to one click. It can be calculated with travelled distance. For example, $193 \mathrm{~m}$ (for 1 meter jump) is equal to 193 clicks. 
Unlike the viewpoint snapping technique, we did not apply any fading animation to avoid the user from being disoriented. The jumping with $416 \mathrm{~ms}$ delay was quite fast. Adding a fading animation would result in the screen constantly blanking, which may be distracting or cause eye strain. 


\section{Chapter: Viewpoint Snapping: User Study 1}

A user study was employed to compare the effectiveness of viewpoint snapping to reduce cybersickness. Its impact on user performance was assessed, as were subjective measures of cybersickness levels and presence. Two groups of participants were compared: one that experience viewpoint snapping (VS), and a control condition group with no visual snapping (NVS). Our main hypothesis is that the use of viewpoint snapping will significantly reduce cybersickness, as compared to a control condition without it.

\subsection{Participants}

A total of 28 participants were recruited. They were between 18 and 35 years of age with a mean age of 26.35 years. There were a total of 17 males and 11 females. the participants were divided into two groups. The first group -5 females and 9 malesexperienced VS condition, while the second group -6 females, 8 males-experienced NVS. The experiment was approved by our university's research ethics board (CUREB-B Clearance \# 107640). With the exception of two participants who wore glasses, all participants had normal vision.

The study recruited participants with a wide range of experience with HMD VR systems: 6 participants had never used VR system before, while 3 participants used VR frequently ( 1 to 6 times per week). Of the remaining participants, 5 had used VR between 1 to 5 times, and the rest had used VR system 6 to 15 times. With regard to cybersickness, 6 participants indicated that they had experienced some level of cybersickness previously. 
The perceived reasons for these experiences ranged from virtual movement when stationary, movement in a flight game, and technical issues like refresh rate, and jitter. None of the participants reported having the flue, taking and nausea-related medicine, or any other similar medical conditions.

\subsection{Apparatus}

Hardware: The experiment was conducted using a PC (i5-6500 3.2GHz CPU 3.2, GeForce GTX 970 GPU, 8GB RAM) with an Oculus Rift CV1 head-mounted display. The input devices included an Eastern-Times Tech gaming mouse (ET7) and a keyboard. According to previous work [18] the mouse offers superior navigation speed, so the current study used it rather than a joystick. The setup is seen in Figure 7. To avoid potential fatigue effects, or potential harm to participants (e.g., falling due to dizziness), participants were always seated. This also reduces any demand for postural controls [37].

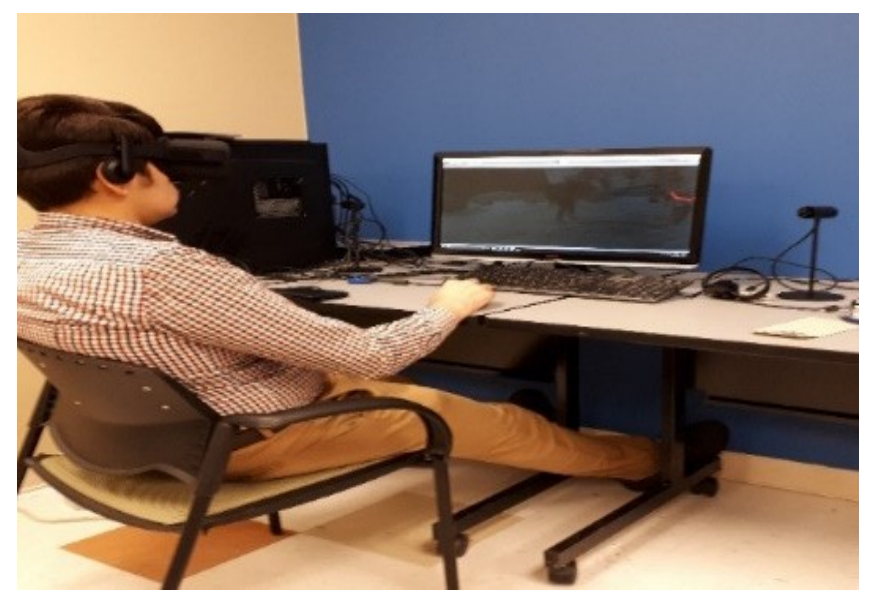

Figure 7 Hardware setup, depicting a participant taking part in the experiment. Participants were seated on a fixed chair to avoid any movement or real body rotation. 
Software: A custom virtual environment in Unity3D was developed (Figure 2), the available FPS-level demo was used as a base (Figure 2), and the game was customized to add data collection and to implement viewpoint snapping. NavmeshAgent were used for the enemies so that they would follow approach the player position, or main camera. NavmeshAgent is available via the Unity Engine AI system. The study used two version of the environment: one with viewpoint snapping, and one without. As is typical of FPS games, the player view vector was coupled with the mouse.

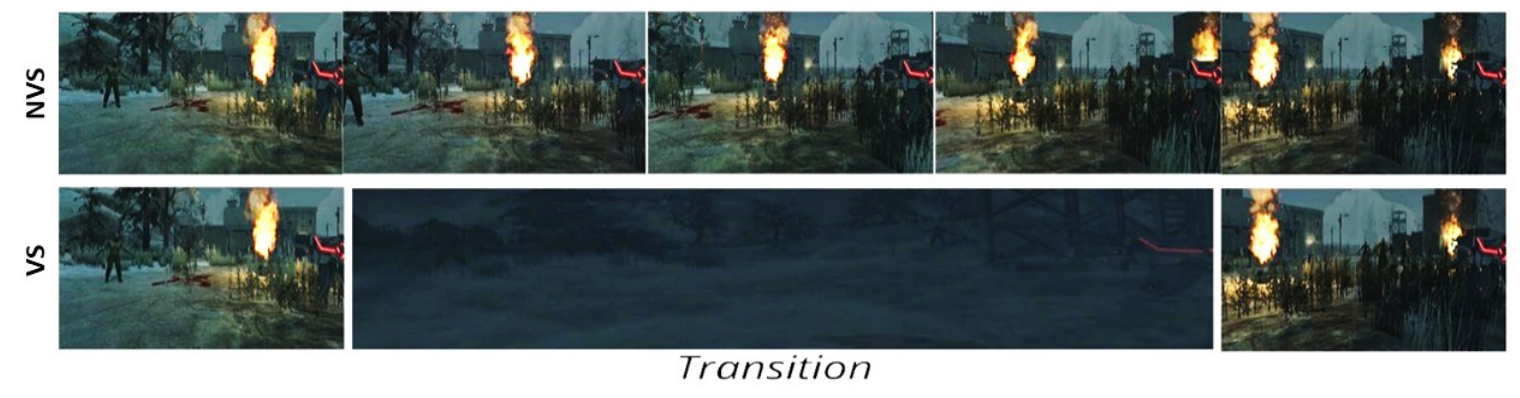

Figure 8 The top row of images shows a standard (non-snapping) viewpoint rotation. The second row shows the same rotation with our viewpoint snapping technique enabled. A fast transition eliminates the intermediate frames: meaning the rotation becomes discrete,

The player stood in the middle of the environment while a stream of 40 zombie enemies approached them. Each stream took approximately 2 minutes to reach the player from their starting points. In total, there were 10 streams of 40 enemies each, or 400 enemies in total. The starting positions of the zombies were consistent from one trial to the next. Since our study focused only on viewpoint, yaw and camera pitch was disabled. Thus, the zombies always appeared in positions where the participant could shoot them without the need to aim up or down. This used ray-casting originating at the participant position. The 
participant avatar was depicted holding a gun, as the task involved shooting the zombies (Figure 9). This was done intentionally to ensure participants did not use their head movement to aim as an excessive head movement could increase the cybersickness level [37]). Movement was disabled, and the participant was always positioned in center of the screen to ensure that translation did not have any effects on the study. Finally, as described earlier, a black fading animation/transition was added during the snapping.

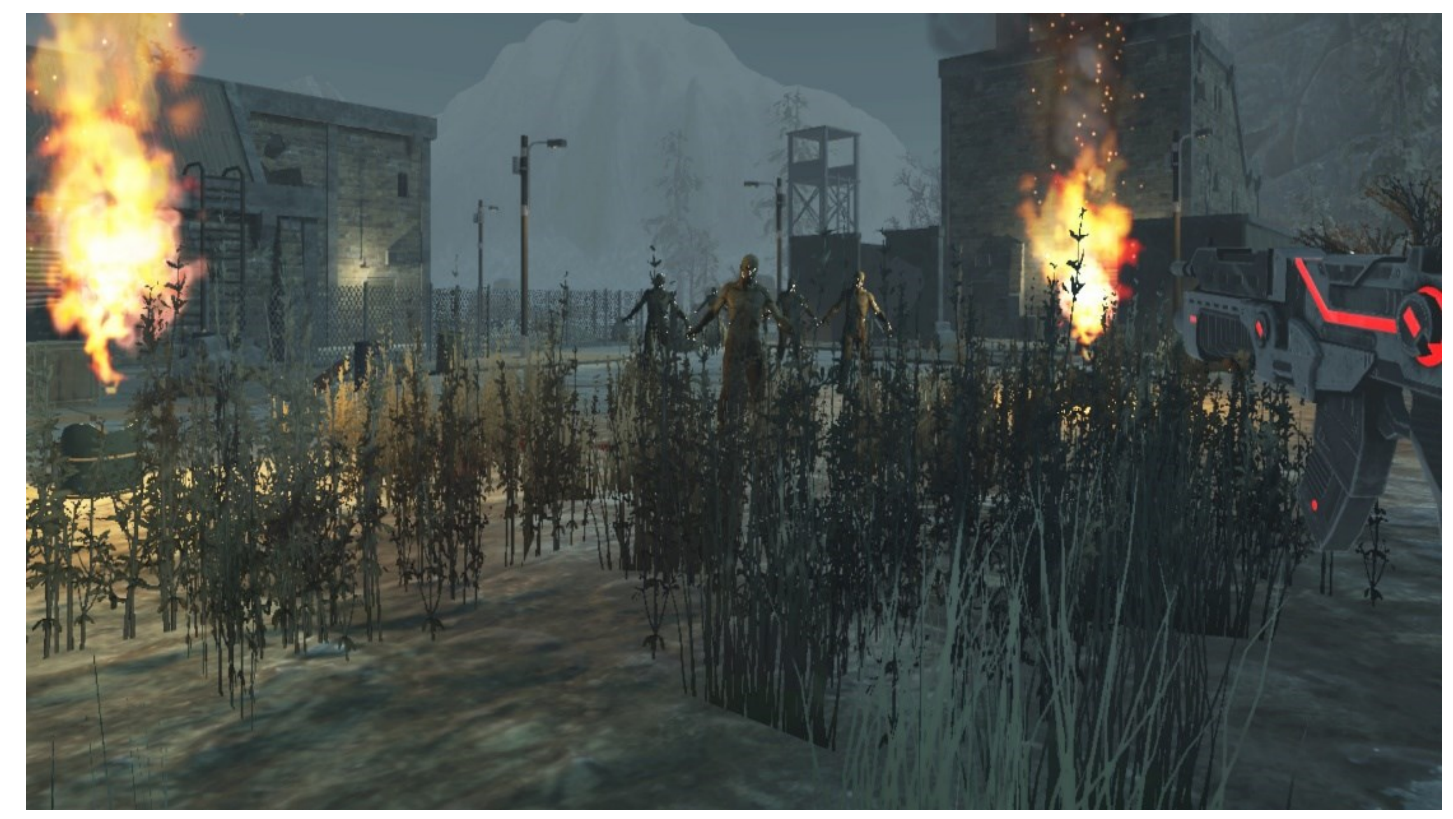

Figure 9 Player View Point

\subsection{Procedure}

Participants first signed a consent form. The experimenter then explained the purpose of the experiment. Participants were informed that they could quit the study at any time, and for any reason, but especially if they felt too nauseous. The experimenter then explained the details of the task. 
The task involved shooting at the zombies that appeared around the participant. To shoot a zombie, the participant had to use the mouse to center the viewpoint on the zombie and then press the left mouse button, much like most mouse-based first-person shooter games. If they successfully clicked/shot the zombie, the zombie would disappear. Zombies were positioned pseudo-randomly and distributed to appear outside of the field of view, necessitating a great deal of rotational viewpoint movement for participants to find and shoot them. Zombies would slowly advance from their starting position to the participant's position. If a zombie came within 3 meters of the participant's position without being shot, they still disappeared, but this was considered a miss/error for the purpose of our performance-based dependent variable.

Like previous studies [13], a nausea questionnaire (Figure 10) appeared on the screen every two minutes, and the participant rated their current nausea level from 1 to 10 . If they gave a score of 10 , they were advised to withdraw from the experiment; three (3) participants withdrew in this fashion. Otherwise, participants performed the task in VR for a total of 20 minutes in either the VS or NVS conditions.

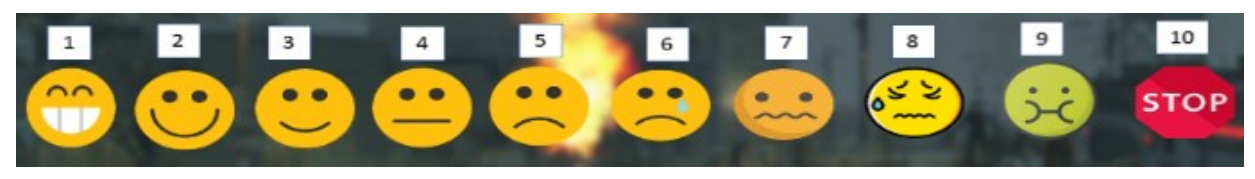

Figure 10 - Nausea Likert scale questionnaire

Participants completed the SSQ questionnaire [31] twice: once before the study (PreSSQ) and once after. Only participants who had a Pre-SSQ score of less than 7.48 were permitted to take part in the study [10]. Nobody will report more than 7.48 in the pre-SSQ questionnaire. They were also asked to sit and rest for 5 minutes before the study to ensure 
any effects from walking or running to study location would dissipate prior to commencing the study.

Following completion of the experiment and the Post-SSQ test, participants also completed the Witmer and Singer presence questionnaire [64]. We then interviewed and debriefed participants. Participants were compensated with $\$ 10$ for their time.

\subsection{Design}

Consistent with past cybersickness studies [33, 55], our experiment employed a between-subjects design, with a single independent variable: viewpoint snapping (enabled: $V S$, or disabled: $N V S)$.

The dependent variables included Total SSQ, Total Presence, error rate (count of trials where a zombie reached the participant), and nausea scores (measured on a 10-point Likert scale, as discussed earlier). The hypothesis states that with viewpoint snapping enabled, levels of cybersickness and nausea will decrease, but the error rate and presence will be significantly worse

\subsection{Results}

\subsubsection{Total SSQ}

Cybersickness was quantified using the Simulator Sickness Questionnaire (SSQ), developed by Kennedy et al [31]. The SSQ consists of 16 symptom categories aggregated into three major components, Nausea (e.g., general discomfort), Oculomotor problems (e.g., eyestrain), and Disorientation (e.g., vertigo). The total score is obtained from subscores calculated in each of these three components. Total SSQ is then calculated as 
$($ Nausea score $)+($ Oculomotor score $)+($ Disorientation score $) \times 3.74$ [31]. Cybersickness was quantified using the SSQ developed by Kennedy et al. [31]. The SSQ consists of 16 symptom categories aggregated into three major components, nausea, oculomotor and disorientation (see Appendices A.1).

Results for total SSQ scores are seen in Figure 11. Overall, viewpoint snapping (VS) did indeed yield lower SSQ scores compared to the non-viewpoint snapping condition with average scores of 29.8 and 48.1 respectively. An independent sample t-test was conducted to compare the differences in total SSQ between the VS and NVS conditions. There was a significant main effect for viewpoint snapping on total SSQ $(\mathrm{t}(26)=2.3, p=0.026$, power $=.79$ ). Viewpoint snapping significantly lowered cybersickness levels compared to the control condition, as measured by total SSQ.

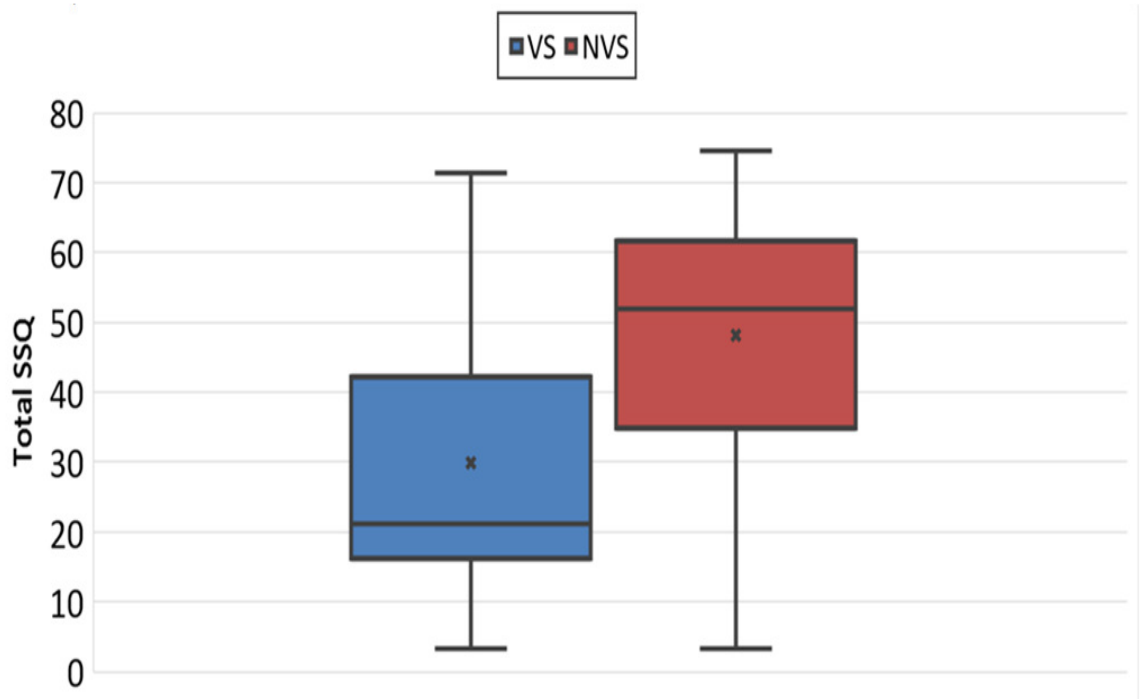

Figure 11 Box plot of total SSQ Scores. Lower Score is better. 


\subsubsection{Nausea Score}

Nausea scores were taken every two minutes. Nausea scores as a function of time are seen in Figure 12.

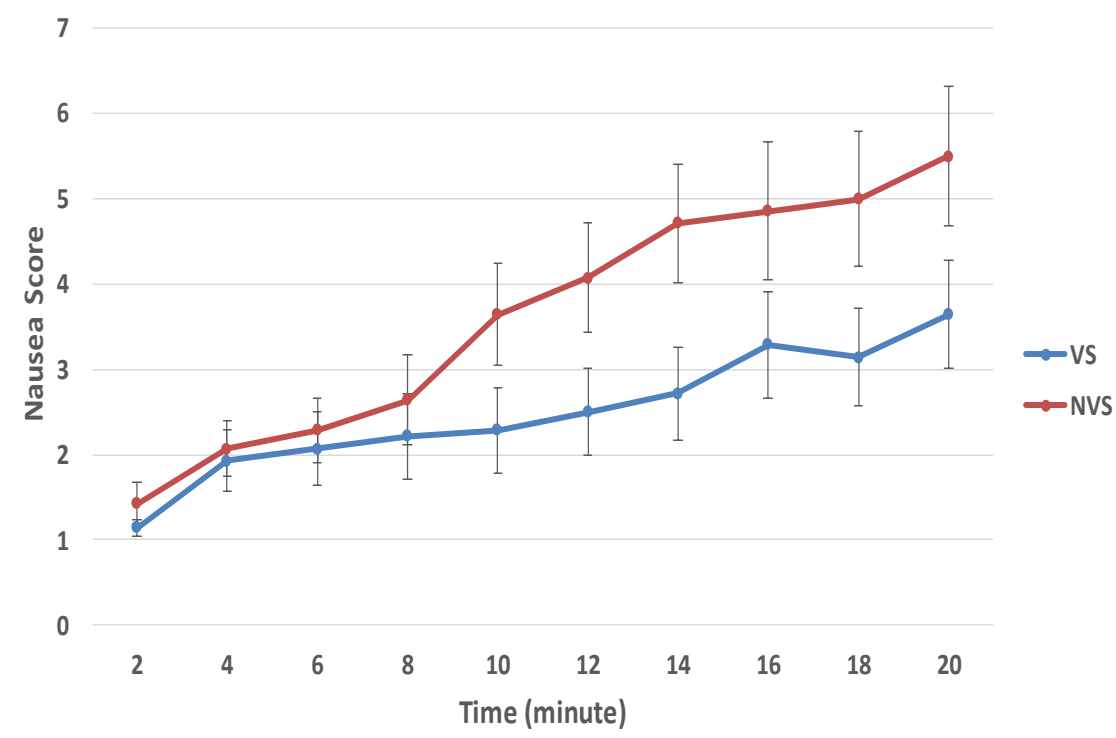

Figure 12 Total nausea differences as a function of time. Error bars show standard error.

Unsurprisingly, nausea levels increased over time due to the excessive viewpoint rotation necessitated by the experimental task. What is interesting is that viewpoint snapping again resulted in reduced symptoms as compared to the non-viewpoint snapping condition. Repeated ANOVA measures were performed to evaluate the effect of viewpoint snapping and exposure time on nausea scores. There was a significant main effect for viewpoint snapping on nausea scores $(F 1,9=20.7, p=0.0012)$. The viewpoint snapping group had significantly lower nausea scores. The effect of time was also significant $(F 9,9$ $=7.8, p=0.0027)$. As seen in Figure 12, nausea scores increased with time. However, the interaction effect between viewpoint snapping and exposure time was not significant $(F 9,9$ $=0.9, p=0.5$ ), suggesting that both viewpoint snapping conditions increased in nausea at 
about the same rate. It is possible that a longer experiment or a larger participant pool may reveal differences as the trends appear to diverge slightly from the 20-minute mark in Figure 12.

\subsubsection{Error Rate}

The total number of errors (the number of times a zombie reached the player) was not significantly different between the two viewpoint snapping conditions (see Figure 13). An independent sample t-test was conducted to compare differences in total error between the VS and NVS conditions. The difference was not significant $(\mathrm{t}(26)=0.3227, p=0.7)$. While this does not categorically demonstrate that error rate is not affected by viewpoint snapping since one cannot "prove the null hypothesis" this way, this could be interpreted as a positive sign that any performance difference due to viewpoint snapping is potentially small.

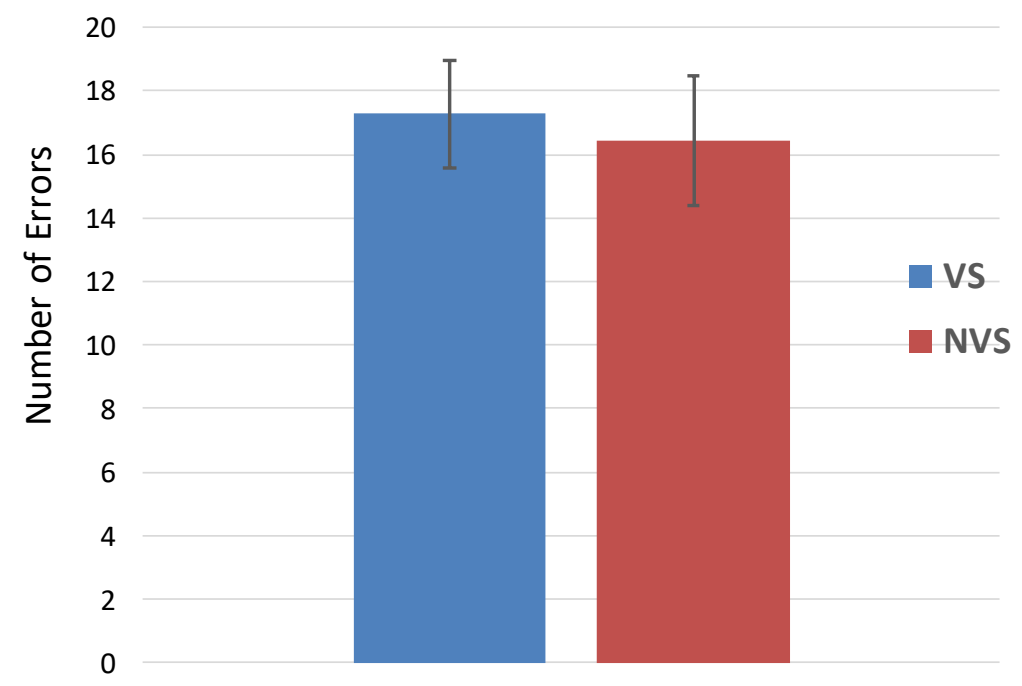

Figure 13 Error rate by viewpoint snapping. Error bars show $\pm 1 S E$. 


\subsubsection{Presence}

Similarly, the result of the 23-question Witmer and Singer presence questionnaire [64] revealed no significant difference between two groups in terms of presence, as confirmed by an independent samples t-test $(\mathrm{t}(44)=1.9, p=0.06)$. Note that presence was slightly (but not significantly) lower for the VS group (mean $=4.16, S D=1.2)$ than the NVS group (mean $=4.89, S D=1.4)$.Similarly, the result of the 23 -question Witmer and Singer presence questionnaire [64] revealed no significant difference between the two groups in terms of presence, as confirmed by an independent samples t-test $(\mathrm{t}(26)=1.9, p$ $=0.06)$. It is important to note that presence was slightly but not significantly lower for the VS group $($ mean $=4.16, S D=1.2)$ than the NVS group $($ mean $=4.89, S D=1.4)$.

\subsection{Discussion}

Overall, viewpoint snapping significantly reduced participant cybersickness levels (per the SSQ) by about $40 \%$. As argued earlier, this makes sense and is consistent with our expectations based on past research on inconsistent camera movement and reducing optic flow $[8,54]$.

Of course, viewpoint snapping introduces a tradeoff between user comfort and naturalism/realism, much like other cybersickness reduction methods, such as blurring, headlock, and field-of-view reduction (or "tunneling"). Interviewing participants after the experiment revealed some insights. For example, 4 participants out of 14 in the VS condition mentioned that they initially found the snapping disoriented them. This may explain why this group had slightly, although not significantly, worse error and presence scores. However, after playing for a few minutes, participants indicated that they eventually 
got used to the snapping and it began to feel more comfortable. For example, one participant indicated that "at the start of the game it was annoying and frustrating to jump to different angles, but after 2 or 3 minutes" the participant "could control her actions better".

The result that presence and error rates were not significantly worse with viewpoint snapping was surprising as it was inconsistent with the hypothesis. This, though, may suggest a limited impact of viewpoint snapping on objective user performance and presence in VR games. Further studies will help gather additional support for (or refute) this result, though, this is not definitive at this time.

Interestingly, two participants did not even notice the snapping occurring during the experiment. We note that both participants had very limited VR experience - one had no prior exposure, and the other only had 1 to 5 prior VR experiences. After the experiment, when asked if they noticed the snapping, both indicated that thought that the snapping feature was part of the game.

We also note that three participants mentioned that the transition animation was distracting. The blinking of the screen made them dizzy and blurred their vision. Two participants mentioned this was particularly true for large rotation angles, which made it more disorienting and harder to aim. That said, our objective error results indicate limited impacts on user performance.

Finally, as mentioned earlier, three participants withdrew from the experiment. We note that one of these withdrew from the VS condition at the 14-minute mark. The other two withdrew from the NVS condition, at the 4- and 7-minute mark, respectively. It is 
noteworthy that these withdrawals occurred more frequently and much earlier in exposure when viewpoint snapping was not enabled. This may indicate that viewpoint snapping can help increase VR exposure time prior to experiencing adverse cybersickness effects; but, this too needs further exploration in the future. Of course, the task we used in our experiment was an extreme example designed to elicit a cybersickness response. 


\section{Chapter: Translation Snapping: User Study 2}

We conducted a second user study to evaluate the effectiveness of translation snapping in reducing cybersickness. Of course, cybersickness was not our sole concern; after all, if the technique decreases cybersickness but yields a major user performance penalty, its benefits would be questionable. As a result, we measure its effects on performance and presence as well [4]. We recruited two groups of participants. One group experienced translation snapping (TS) in the first session, and standard movement i.e., no translation snapping (NTS) in the second session. The other group experienced both conditions in the reverse order (first NTS and then TS). Our main hypothesis were:

- H1: Employing translation snapping will significantly reduce cybersickness, both in terms of SSQ scores, and subjects' nausea levels.

- H2: Participants experiencing TS in the first session will experience lower cybersickness levels in the second session (NTS) than participants experiencing NTS in their first session. We suspect this asymmetric transfer effect may occur if translation snapping helps "train" participants to be less susceptible to cybersickness, even when it is later disabled.

- H3: Presence, task performance, and subjective preference score will be significantly better with NTS, than with TS. 


\subsection{Participants}

We recruited 20 participants aged between 18 and 39 years (mean age of 28.7 years, 14 male, 6 female). Participants were divided into two gender and age-balanced groups. The first group experienced TS condition in the first session, and NTS in the second session. The second group experienced the conditions in the opposite order. The experiment was approved by our university's research ethics board (CUREB-B Clearance \# 107640). With the exception of four participants who wore glasses, all participants had normal vision.

We recruited participants with a wide range of experience with HMD VR systems: 3 participants had never used VR system before, while 2 participants used VR frequently (1 to 6 times per week). Of the remaining participants, 9 had used VR between 1 to 5 times, and the rest had used VR system 6 to 15 times. With regard to cybersickness, 7 participants indicated that they had experienced some level of cybersickness previously. None of the participants reported having the flu, taking and nausea-related medicine, or any other similar medical conditions. Ten participants did not consider themselves susceptible to motion sickness [26], but seven participants reported that they were slightly susceptible to motion sickness. Two participants considered themselves moderately susceptible and one participant believed that he was very prone to cybersickness in particular.

\subsection{Apparatus}

\subsubsection{Hardware}

The experiment was conducted on an ASUS (Model: GL502VS) gaming laptop (i76700 HQ 2.6GHz CPU 2.59 GHz, GeForce GTX 1070 GPU, 32GB RAM) with an Oculus 
Rift CV1 head-mounted display with 2 tracking cameras. We used the Oculus touch controllers as input devices (see Figure 14).

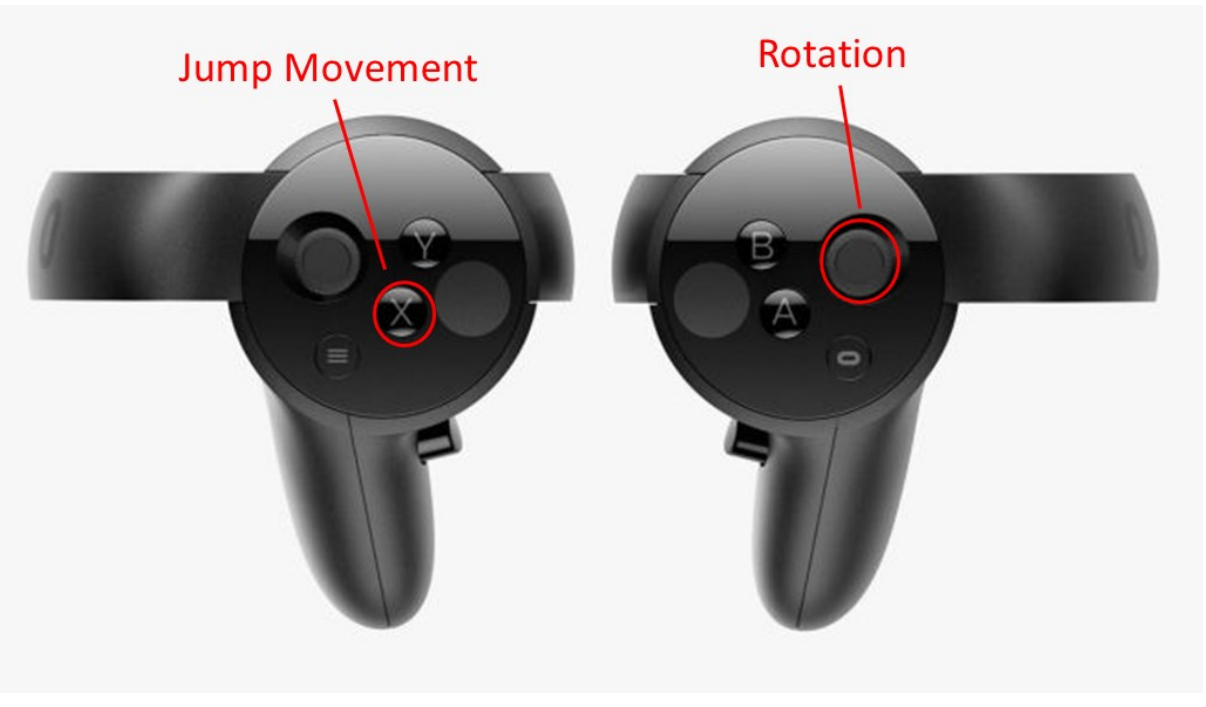

Figure 14 - Oculus touch controller and its controller mapping.

To move forward, participants would hold the "X" button. Holding this button caused the viewpoint to jump forward in $1 \mathrm{~m}$ increments, every $416 \mathrm{~ms}$. These thresholds were determined via our preliminary experiment, as detailed in Section 3.2. Viewpoint rotation (which also controlled movement direction) was controlled using the right thumbstick, consistent with many first-person video games. The setup is seen in Figure 15. To avoid potential fatigue and also reducing any demand for postural controls, the participants were seated [37]. 


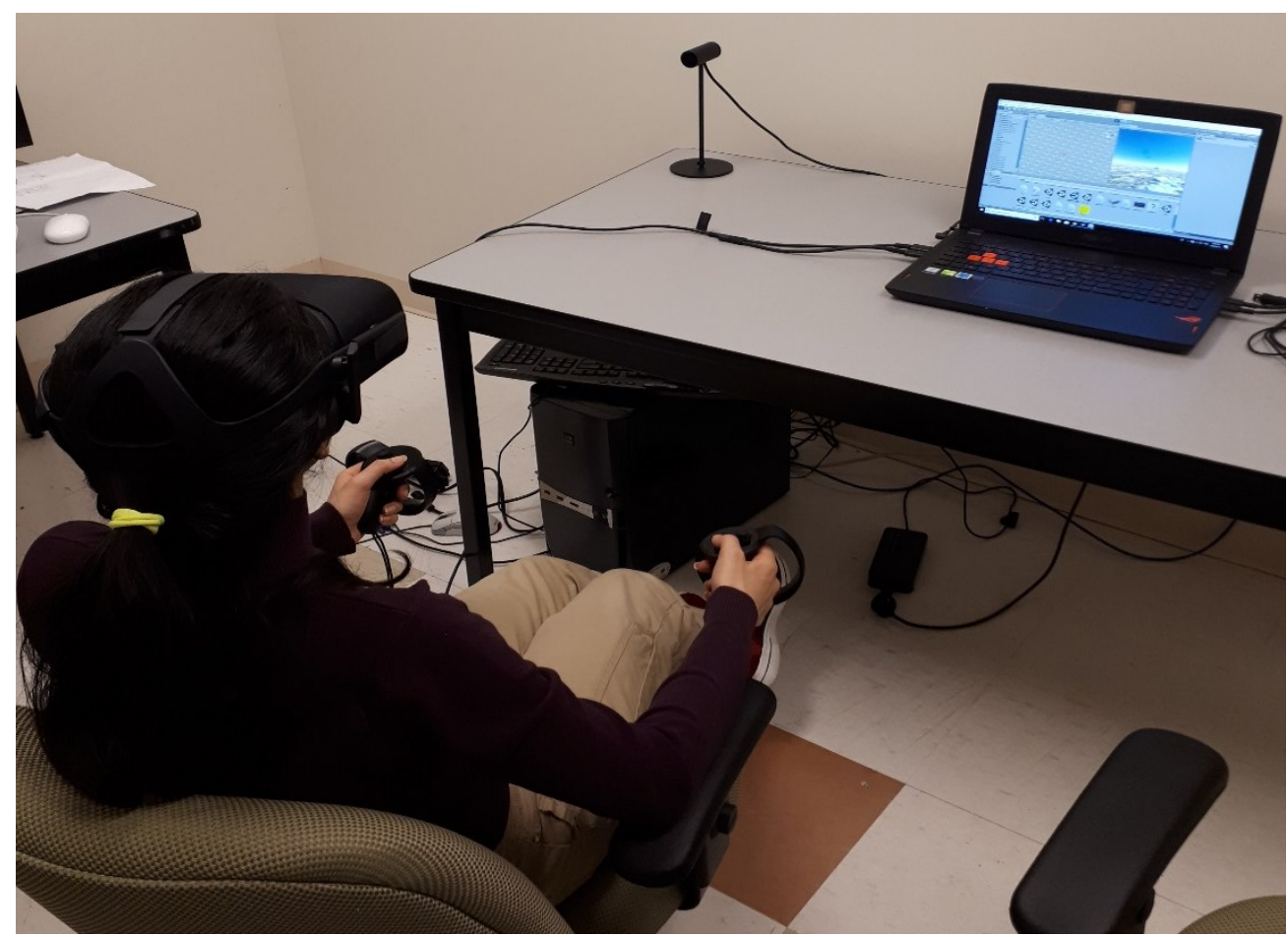

Figure 15 Hardware setup, depicting a participant taking part in the experiment. The chair was locked to avoid real body rotation.

\subsubsection{Software}

We designed a virtual environment for our study using the Unity 3D game engine (version: 2017.1.1f1). To avoid participant distractions, we removed all objects and landmarks from environment terrain. The ground was flat and used a desert-like texture that uniformly repeated over the ground. The software also displayed 10 bright green cones and 8 blue cones that served as waypoints, and a red circle showing the starting position. The environment is depicted in Figure 16. 


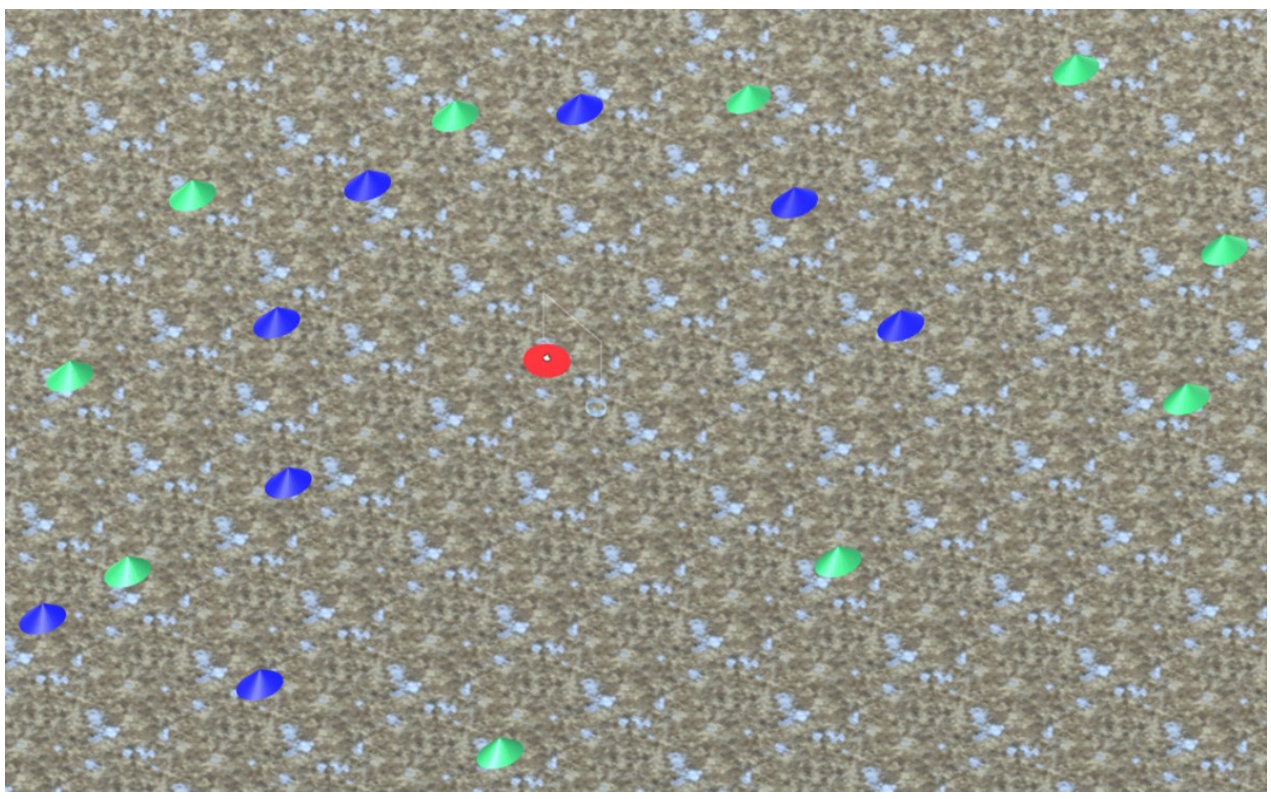

Figure 16 - Overhead view of virtual environment and waypoints - The green cones served as single waypoints and the blue cones served as double waypoints (i.e., the participant must travel to 2 blue cones in these trials).

\subsubsection{Navigation Task}

The task involved navigating from the starting position (red circle) to the green and blue cones, and was modeled after a path integration task used in previous work $[2,40]$. The secondary purpose of this task was to determine if translation snapping negatively impacted participant spatial awareness, as reflected in their ability to determine their original starting point upon reaching a specific waypoint.

At the start of each navigation trial, participants were positioned on the red circle (start location). A single cone would be active and visible - all other cones were initially deactivated (invisible). Participants were instructed to move to the active cone by rotating their viewpoint to control their movement direction, then holding the " $\mathrm{X}$ " button as 
described above to move forward (regardless if they were in the TS or NTS condition). If the cone was blue, the participant had to travel to two cones (first the visible one, then a second one that appeared upon reaching it) in the order they appeared on the ground. Upon reaching a green cone or second blue cone, both the cone and the starting red circle would disappear. In this fashion, on each trial, participants completed either a "single waypoint" task (green cone trial), or a "double waypoint" task (two blue cones trial). This was intended to further assess the possible loss of spatial awareness due to translation snapping.

In total, there were 18 waypoint cones -10 green and 8 blue - located around the start position in different distances. In total 14 trials were recorded for each session. Participants first finished two single waypoints trials (S), followed by one double waypoint trial (D), and repeated this ordering yielding a total of 10 single waypoint trials, and 4 double waypoints trials per session ( $\mathrm{SSD}, \mathrm{SSD}, \mathrm{SSD}, \mathrm{SSD}, \mathrm{SS})$. This task ordering prevented participants from memorizing the path by repeating single waypoint trials repeatedly; double waypoints would increase the need for spatial awareness in the next part of the task, which involved pointing back at the starting location

Upon completing a trial, a message appeared on the screen, asking the participant to point back at the original start position. Participants were instructed to point the Oculus Touch controller at where they thought the starting circle originally was. The position was determined by using ray-casting: a blue ray cast laser was emitted from the right Oculus Touch controller, and a cursor was drawn where it intersected the ground. See Figure 17. Upon pressing the left trigger button, the pointing error dependent variable (see below for 
details) was calculated and the participant was then instantly positioned back at the start location for the next trial.

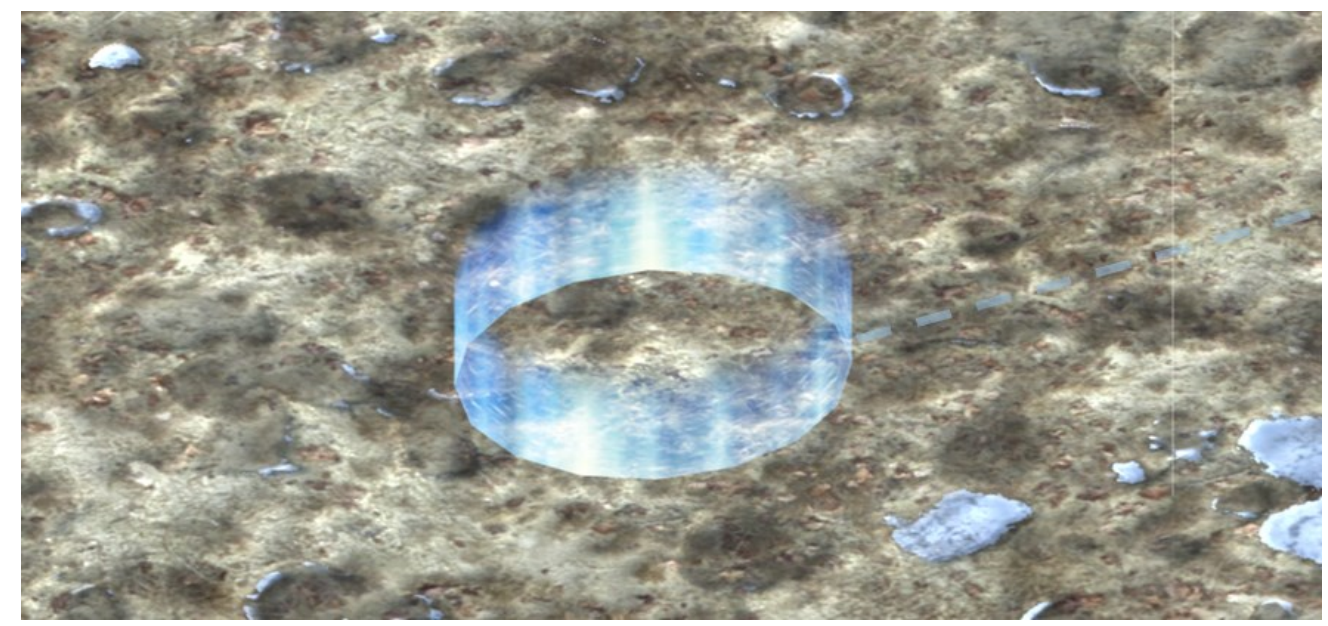

Figure 17 - Cursor as an indicator for users' teleport point

The purpose of using this "teleportation" sub-task was to provide a measure how well participants could perform path integration during translation snapping. It allowed us to quantify how the TS or NTS conditions affect user ability to correctly rotate to the start location, and how close to the start they could get. After all, poor path integration is a possible negative side effect of translation snapping. We called this metric pointing error rate ( $\mathrm{PE}$ for short). $\mathrm{PE}$ was calculated as the distance between the user selected point to the actual original starting point. The task (both for single waypoint green cones and double waypoint blue cones), as well as a depiction of PE, are seen in Figure 18. 

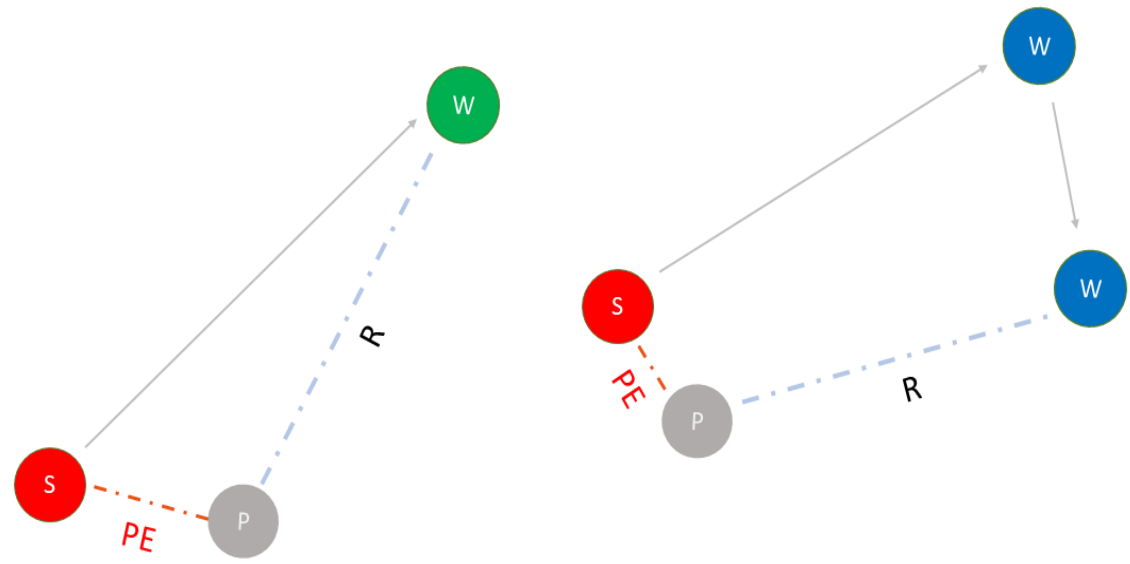

Figure 18 - (Left) Single waypoint trial: The participant starts at the red circle (start position) and travels to the green circle. $R$ is the ray cast line and $P$ is the point selected by participants. The distance between $P$ and the start position is PE (Pointing Error). (Right) Double waypoint trial: The participant starts at the red circle and moves to the first blue waypoint, then the second one, in sequence. Participants performed the point selection task after reaching the second waypoint. PE is calculated in the same fashion as single waypoint trials.

\subsection{Procedure}

Participants first signed an informed consent form. The experimenter then explained the purpose of the experiment. Participants were informed that they could quit the study at any time, and for any reason, but especially if they felt too nauseous. The experimenter then explained the details of the task. The study run in 2 sessions, separated by a 10 to 48 -hour gap. Participants were compensated \$10 upon completion of the second session.

First, we asked participants to fill demographic forms and pre-SSQ questionnaire to ensure they do not feel any symptoms before the study. If their Total Sickness was greater than $7.48[10]$ we asked them to rest for 5 minutes or postpone the study. All participants had scores lower than this. Afterward, we explained the task, the controls and then we 
asked them to practice the waypoint travel task for 2 minutes. Practice trials were not recorded. Participants only completed the practice trials on their first session, since by the second session, they were already familiar with the apparatus and task.

Participants performed the waypoint travel task as described in section 5.2.1 with both the TS and NTS conditions, one condition per session. Condition order was counterbalanced with half of the participants completing TS in the first session, followed by NTS in the second session, and the other half in the opposite ordering.

Every minute, a nausea questionnaire (Figure 19) appeared on the screen, and the participant rated their current nausea level from 1 to 10 , similar to previous studies [13]. If they gave a score of 10, we advised them to withdraw from the experiment; two (2) participants withdrew in this fashion. Otherwise, participants performed the task in VR for around 12 minutes in total, in either the TS or NTS conditions.

Following completion of the experiment and the Post-SSQ test, participants also completed the Witmer and Singer presence questionnaire [64] and NASA TLX [20] questionnaire. We then interviewed and debriefed participants. 


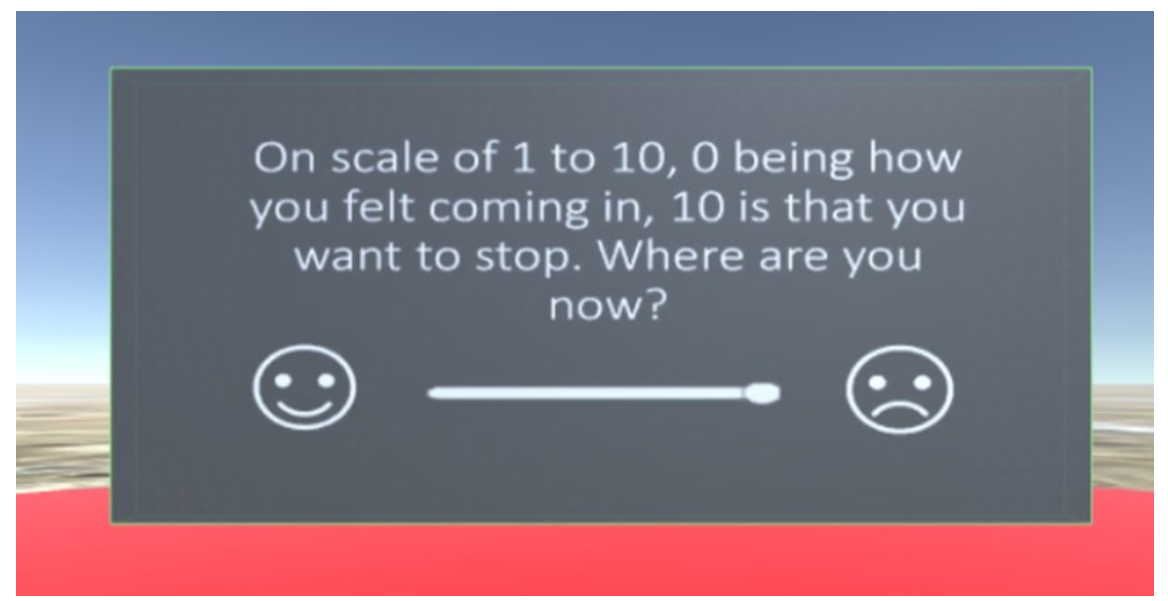

Figure 19 - Nausea (discomfort) Likert scale on-screen questionnaire.

\subsection{Design}

Our experiment employed a within-subjects design with a single independent variable: translation snapping (enabled: TS or disabled: NTS).

The dependent variables included Total SSQ, Total Presence, Pointing Error (PE, calculated as described earlier), and nausea scores (measured on a 10-point Likert scale, as discussed earlier).

\subsection{Results}

As two participants withdrew from the study in NTS conditions, we removed their result for calculating PE, presence, and NASA TLX scores. We kept their results for post-SSQ scores and used their last nausea score before their withdrawal [55]. 


\subsection{1}

\section{Total SSQ}

Results for total SSQ scores are seen in Figure 20. Overall, TS resulted in lower SSQ scores compared to NTS, with average scores of 27.1 and 52.1 respectively. An independent sample t-test was conducted to compare the differences in total SSQ between the TS and NTS conditions. There was a significant main effect for translation snapping on total SSQ $(\mathrm{t}(38)=2.09, p=0.021$, power $=0.81)$. Translation snapping significantly lowered cybersickness levels compared to the control condition (without snapping), as measured by total SSQ.

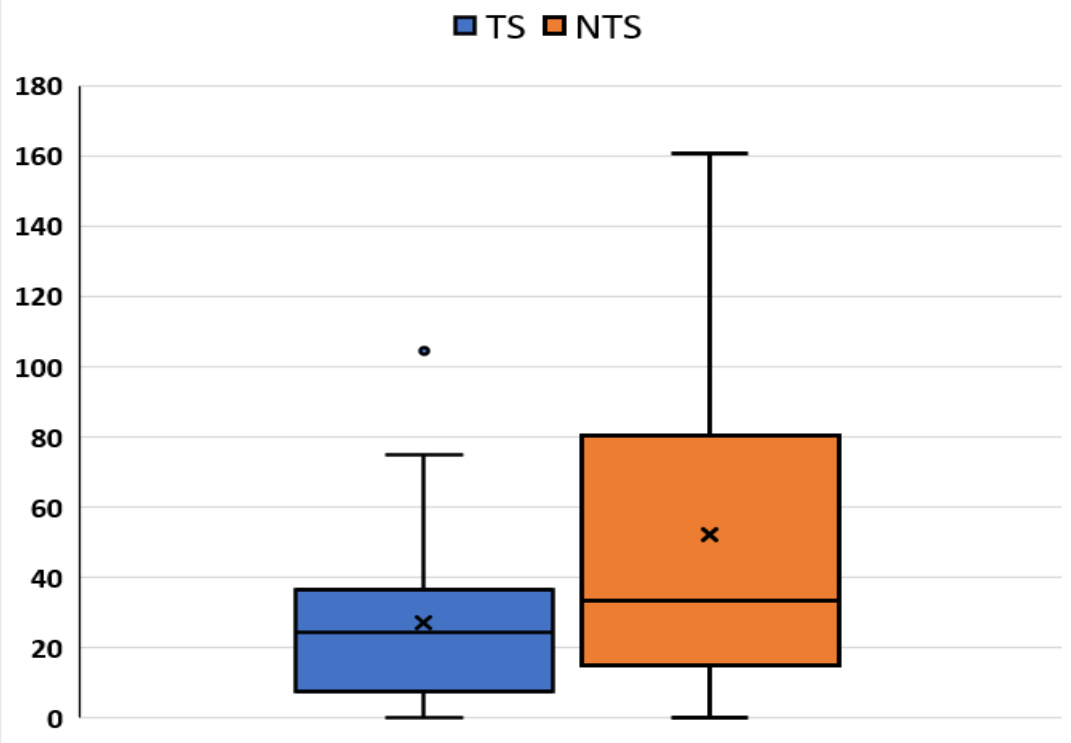

Figure 20 -Box plot of total SSQ Scores. Lower Score is better.

\subsubsection{Nausea Scores}

Nausea scores were taken once per minute. Nausea scores as a function of time are seen in Figure 21. Unsurprisingly, nausea levels increased over time due to exposure increasing effects cybersickness effects [32]. What is interesting is that translation snapping resulted 
in reduced symptoms as compared to the NTS condition. Repeated measures ANOVA was performed treating translation snapping and exposure time as factors. There was a significant main effect for translation snapping on nausea scores $(F 1,19=5.481, p=0.03)$. TS offered significantly lower nausea scores. The effect of time was also significant $(F 9,171$ $=13.96, p<0.0001)$. As seen in Figure 21, nausea scores generally increased with time in both the NTS and TS conditions. However, there was a significant interaction effect between translation snapping and exposure time $(F 9,171=5.04, p<0.0001)$. As can be seen in Figure 21, the two conditions increased in nausea at different rates, diverging quite strongly after about 6 minutes of exposure.

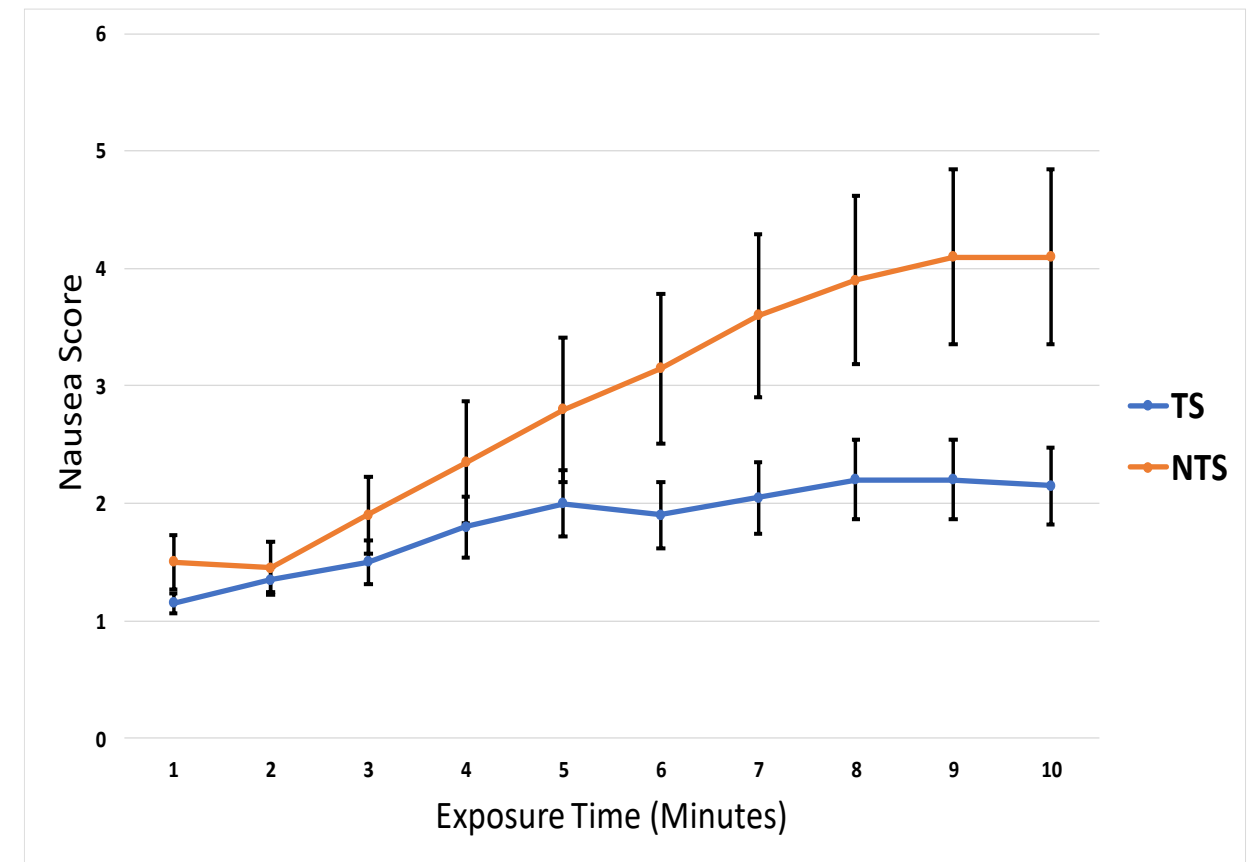

Figure 21 Average nausea scores as a function of exposure time. Error bars show standard error. 


\subsubsection{Average Pointing Error}

Average pointing error (PE) was calculated as explained in section 5.2.1. Average PE scores are seen in Figure 22. An independent sample t-test was conducted to compare differences in pointing error between the TS and NTS conditions. The difference was not significant $(\mathrm{t}(34)=0.4, p=0.6, n=18)$. Although both conditions reveal relatively poor performance for average pointing error (at around $32 \mathrm{~m}$ from the original starting point), TS did not do significantly worse than NTS, suggesting that the technique may not affect path integration that much.

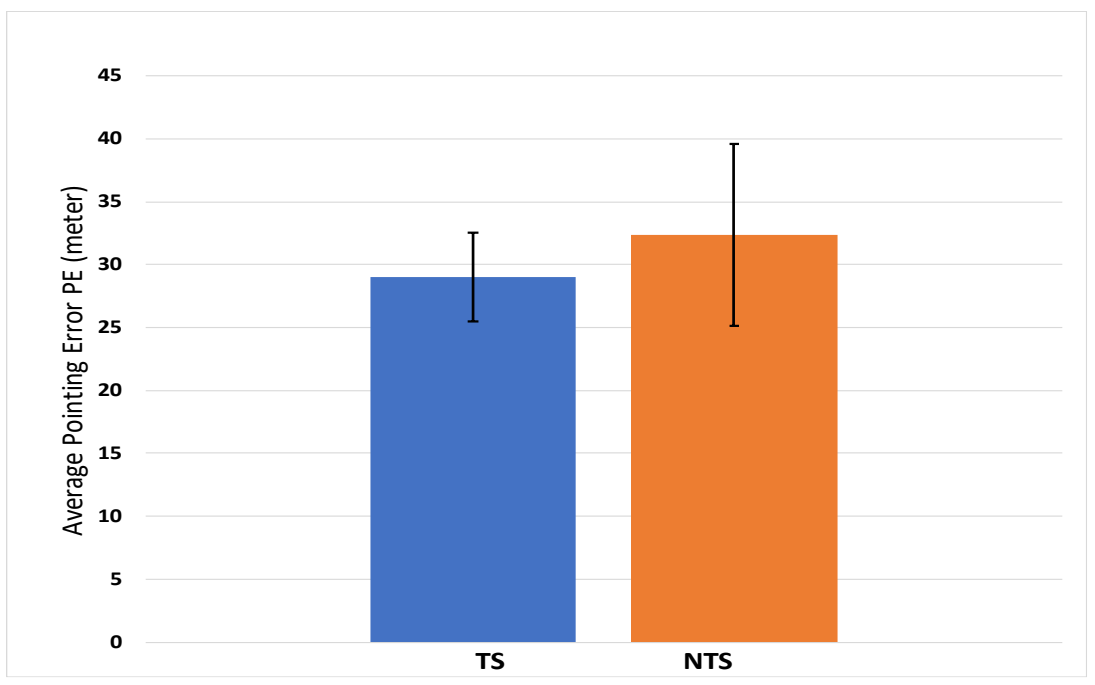

Figure 22 Average Pointing Error (PE) by translation snapping. Error bars show $\pm 1 S E$.

\subsubsection{Presence Score}

We used a customized 16-question version of the Witmer and Singer [64] presence questionnaire, selecting the subset of questions mostly related to our study. Presence scores were not significantly different between the conditions, as confirmed by an independent 
samples t-test $(\mathrm{t}(34)=0.17, p=0.8)$. Note that presence was slightly higher for TS $(\mu=$ 4.2, $S D=2.1, n=18)$ than NTS $(\mu=4.1, S D=2.2, n=18)$.

\subsubsection{Total SSQ between sessions}

Our second hypothesis $(\mathrm{H} 2)$ was that there would be a significant ordering effect on cybersickness. Specifically, participants who experienced TS in the first session and NTS in the second session (henceforth, the TS-NTS group) would have lower SSQ scores in the NTS condition, as compared to NTS scores for participants who experienced NTS first and TS second (henceforth, the NTS-TS group). In other words, our hypothesis was that exposure to TS in the first session would help "train" participants to be less susceptible to cybersickness in the second session, when translation snapping was disabled.

Repeated measure ANOVA revealed no significant difference between the TS-NTS and NTS-TS groups $\left(F_{1,9}=.07, p=0.8\right)$, but there was a significant difference between TS and NTS conditions $(F 1,9=4.1, p=0.04)$. Also there is no interaction effect for TS between two groups $(F 1,9=.003, p=0.9)$.

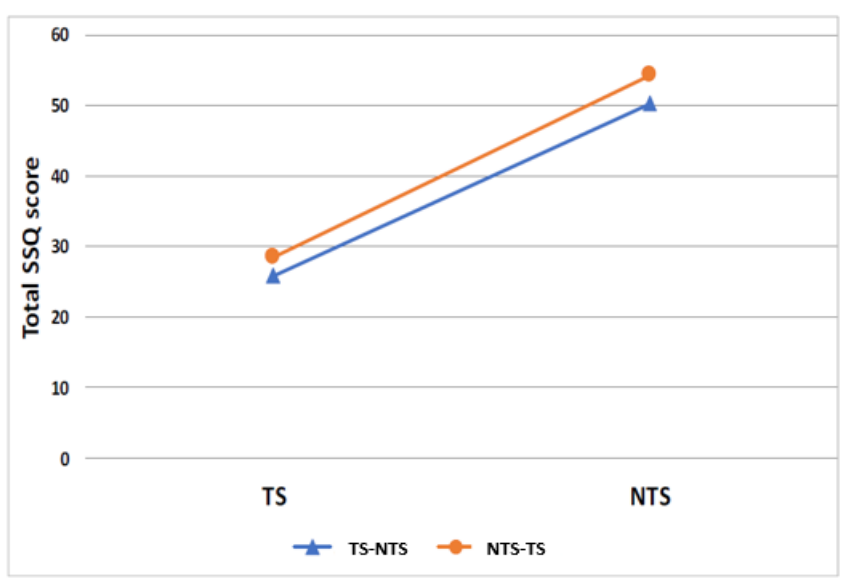

Figure 23 Total SSQ score between 2 groups TS-NTS and NTS-TS. 
As a result, we cannot reject the null hypothesis; TS did not seem to help train participants to become less susceptible to cybersickness.

\subsection{6}

\section{NASA TLX score}

The mean $(\mu)$ and standard deviation $(\sigma)$ scores of six sub-scales of NASA-TLX are seen in Table 5.

\begin{tabular}{lll}
\hline & \multicolumn{1}{c}{ TS } & NTS \\
\hline Mental Demand & $\mu=10.9, \sigma=5.3$ & $\mu=11.2, \sigma=5.6$ \\
\hline Physical Demand & $\mu=6.15, \sigma=6.2$ & $\mu=6.25, \sigma=6.5$ \\
\hline Temporal Demand & $\mu=5.1, \sigma=4.6$ & $\mu=5.2, \sigma=4.8$ \\
\hline Performance & $\mu=12.8, \sigma=5.8$ & $\mu=12.9, \sigma=5.8$ \\
\hline Effort & $\mu=8.4, \sigma=6.7$ & $\mu=8.5, \sigma=6.8$ \\
\hline Frustration & $\mu=4.15, \sigma=4.9$ & $\mu=4, \sigma=4.9$ \\
\hline
\end{tabular}

Table 5 - Nasa TLX scores

The score of physical, mental and temporal demand shows TS has slightly lower demand in comparison with NTS method. In terms of performance, the NTS was slightly better in the selected score. An independent-samples t-test was conducted to compare the overall Nasa TLX score between the TS with NTS conditions. There was no significant difference in between TS $(\mu=7.9, S D=6.3, n=18)$ and NTS $(\mu=8, S D=6.5, n=18)$; $\mathrm{t}(238)$ $=0.11, p=0.9$.

\subsection{Discussion}

Overall, much like viewpoint snapping, and consistent with our expectations based on previous work $[8,54]$, translation snapping significantly reduced participant cybersickness levels (per the SSQ) by 47\%. Like viewpoint snapping, translation snapping also appeared 
to have minimal impact on presence scores. Participants also did not report breaks in presence due to snapping. Unlike the previous study (chapter 4) none of the participants reported any disorientation due to the jumping movements. There are two possible explanations for this. First, TS did not use any fading animation, unlike viewpoint snapping. Hence, the user may feel a less flashy animation can reduce eye strain, since the animation would be playing much faster (every $416 \mathrm{~ms}$ ) in this experiment than with viewpoint snapping. A second possible reason is that we took greater care to "calibrate" the jumping distance based on user preference, unlike viewpoint snapping, which was optimized to reduce nausea.

Six participants reported they felt noticeably more nausea with rotation, than with translational movement. These comments were unsurprising; after all, we did not use any rotation-based technique like viewpoint snapping [17] or tunneling [19]. We note that since both conditions used the same mechanism for rotation, this effect was equal in both the TS and NTS conditions.

Two participants withdrew from the study (one in the first session, another in the second session), both during the NTS session. The first participant noted that they felt nauseous at the end of TS condition, but did not withdraw in that condition. The other participant finished TS condition without reporting any symptoms. Both participants rested for 15 minutes immediately following withdrawal from the study. It is notable (if unsurprising) that both of these withdrawals occurred during the NTS condition.

A few participants suggested changing controller mapping for the teleportation sub-task. For example, they suggested using the trigger button on the same controller for 
teleportation. Two participants also suggested they would prefer to use the thumbstick for movement, rather than using $\mathrm{X}$ button. We will consider this suggestion for future studies. 


\section{Chapter: Conclusion}

\subsection{Summary}

In this thesis, we proposed a method for reducing cybersickness caused by visualvestibular conflicts in stationary VR setups. This method was motivated by previous research on discrete movement and inconsistent locomotion in order to reduce vection or illusionary of self-motion in virtual reality environments.

The first technique is designed based on discrete rotational movement and called viewpoint snapping (VS). The viewpoint snapping technique is designed based a preliminary experiment, assessing discomfort levels of 12 participants at 11 different rotational speeds. The result of this study provided a threshold for activating viewpoint snapping, to reduce cybersickness when users feel the highest level of discomfort. We also add a black fading transition between viewpoints to hide these discrete movements. For evaluating viewpoint snapping we ran a user study with 28 participants in two different groups (14 participants in each group) in the FPS game scenario. We compare viewpoint snapping with controlled condition (without viewpoint snapping) and Total SSQ score, nausea score, presence and error rates (missed targets) were measured. The result indicates that, viewpoint snapping can decrease SSQ-reported cybersickness by as much as $40 \%$ and can also reduce nausea score, especially with longer exposure time. In addition, the results of the study showed the presence level and error rate were not significantly affected by VS technique. 
The second technique, translation snapping (TS) was based on reducing vection during translational movements by jumping movement. Like the previous technique, we ran a preliminary experiment to evaluate four different jumps distances. The outcome of this study gave us a threshold for the most comfortable jump distance, as well as an appropriate speed with which to activate it (e.g., during continuous button/joystick pressing). To evaluate translation snapping we ran a user study with 20 participates in two different sessions, comparing TS to a control condition (without translation snapping). This study was split into two sessions, where participants experienced each condition on different days. Total SSQ score, nausea score, presence, NASA TLX score (physical and mental demand of tasks) were recorded. The task involved a path integration scenario, to help determine if discrete motion negatively affected participant spatial awareness (and hence wayfinding ability). Much like the results of the viewpoint snapping experiment, the results of this study reveal that translation snapping is also effective at decreasing cybersickness. SSQ scores decreased by around $50 \%$ when translation snapping was enabled. Translation snapping also significantly reduced nausea scores, especially with longer exposure time. Moreover, the results of the study showed that presence levels, NASA TLX scores, and pointing error were not significantly affected by the TS technique.

In conclusion, both techniques reduced cybersickness, with potentially low cost in terms of user performance and presence. Moreover, both techniques yielded a notable reduction in nausea levels reported by participants. Overall, our results are promising and motivate us to further study and the effects of combined viewpoint and translation snapping in VR environments. 


\subsection{Limitations and Future Work}

As discussed earlier in Section 3.2.1 there is a potential confound in our viewpoint snapping preliminary experiment, from which we derived our rotational speed threshold at which to activate viewpoint snapping. As argued in that section, we suspect the impact of this potential confound is quite small. For our purpose, the $25 \%$ s threshold appeared to be effective - our viewpoint snapping condition did reduce cybersickness, as expected. Nevertheless, future work will focus on establishing more reliable thresholds, since it is possible our current implementation unnecessarily activates viewpoint snapping at lower rotational speeds than is strictly necessary. A more well-established snapping threshold may yield better results.

Another limitation of the viewpoint snapping evaluation is the fact that participants were stationary while performing the zombie shooting task. Testing the effectiveness of viewpoint snapping during free-roaming navigation is a clear opportunity for future studies and would allow us to evaluate the effectiveness of viewpoint snapping in more realistic tasks. Other topics for future work include determining an empirically validated snapping range. We used $22.5^{\circ}$ in the current experiment based on informal pilot testing. It is likely that "fine-tuning" this parameter by testing different snapping ranges (potentially dependent on rotational speed) will yield better results.

In the translation snapping study, when participants withdrew we used their last score (which was near to 10) as their selected score for remaining minutes. As in the study of cybersickness, the time of the exposure is more important than a task. We have to consider 
raw discomfort score which used by Feiner and Fernandes [13]. This score allows us to calculate nausea score with respect to time. It will normalize the score and shows that a person who withdraws the study soon, should not get high nausea score. In other words, the measured scores from a person who finishes the study should be more significant.

As revealed by the analysis of pointing error, there is no significant difference between TS and NTS. Participants were equally (roughly) able to point back to their initial starting position with both TS and NTS. This further suggests that TS does not yield substantially worse path integration than NTS. This is further supported by participant comments around their apparent lack of disorientation (as compared to viewpoint snapping).

A clear topic for future work is to include both viewpoint snapping and translation snapping in a single experiment. For example, translation snapping can be employed for linear movement, both with and without viewpoint snapping. Such an experiment would determine which of these is more effective in reducing cybersickness, in a more naturalistic VR navigation task.

We also may redesign both translation and viewpoint snapping technique with respect to the speed of camera or rotation. As in the current study, we only find a threshold which may not work in different scenarios. 


\section{Appendices}

\section{Appendix A}

\section{A.1 Measuring SSQ}

The SSQ consisted of 16 questions and each question has a 4-point Likert scale (0none; 1-slight; 2-moderate; 3-severe). According to Kennedy et al. [31] only questions that get score 1 to 3 could be significant for measuring sickness. Beside Total sickness, the SSQ has 3 sub-score categories (Nausea, Oculomotor, and Disorientation). The symptoms and their weights are listed in the following table.

\begin{tabular}{cccc|c}
\hline & \multicolumn{3}{c}{ Weight } \\
\cline { 2 - 5 } SSQ Symptom & $\mathrm{N}$ & $\mathrm{O}$ & $\mathrm{D}$ & $\mathrm{T}$ \\
\hline General discomfort & 1 & 1 & 2 \\
Fatigue & & 1 & & 1 \\
Headache & 1 & & 1 \\
Eyestrain & 1 & 1 & 1 \\
Difficulty focusing & & 1 & & 2 \\
Increased salivation & 1 & & 1 \\
Sweating & 1 & & 1 & 1 \\
Nausea & 1 & & 1 & 2 \\
Difficulty concentrating & 1 & 1 & 1 & 2 \\
Fullness of head & & & 1 & 1 \\
Blurred vision & & 1 & 1 & 1 \\
Dizzy (eyes open) & & & 1 & 1 \\
Dizzy (eyes closed) & & & 1 \\
Vertigo & 1 & & \\
Stomach awareness & 1 & & \\
Burping & 1 & & 1 \\
\hline
\end{tabular}


"Each score is obtained by summing up the product of the severity score and the corresponding weighting for every symptom, and then multiplying by a scaling value (3.74 for total score $(\mathrm{T}), 9.54$ for nausea $(\mathrm{N}), 7.58$ for oculomotor $(\mathrm{O})$, and 13.92 for disorientation (D))" [10]. As you can see some symptoms scores (Like General discomfort) has weight 2 , when we want to calculate them for Total SSQ score, we first need to multiply each user's answer to the weights. For example, if participants assign score 3 to general discomfort we first multiply this number by 2 and then sum all the scores with each other.

The score for nausea is between 0 to 200 , for oculomotor would be between 0 to 159, for disorientation scale range from 0 to 292 and for total SSQ score the scale would be between 0 to 235 . 


\section{Appendix B}

This section is allocated to forms and materials we used for the user study.

\section{B.1 Consent Form for Viewpoint Snapping Study}

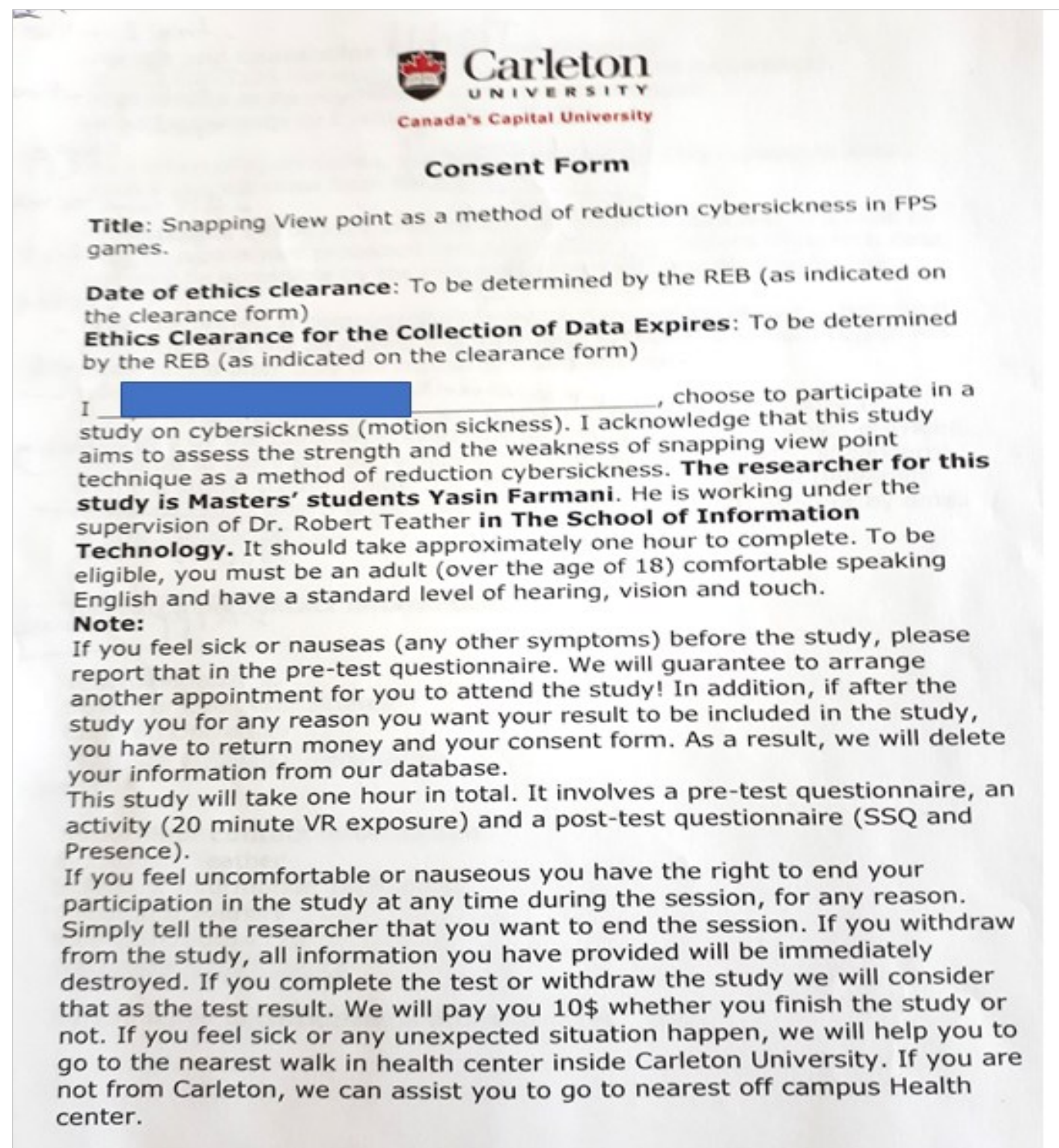


Health and Counselling Services (on campus):

Suite 2600-1125 University Dr, Ottawa, ON K1S 5 B6 on campus).

Meadowlands Family Health Centre (off campus):

888 Meadowlands Dr E, Ottawa, ON K2C 3R2.

As a token of appreciation, you will receive a $\$ 10$. This is yours to keep, even if you withdraw from the study.

All responses will be kept anonymous. All research data and notes will be kept on a password protected computer of the researchers. Research data will only be accessible by the researchers and the research supervisor.

Once the project is completed, all research data will be securely destroyed. Electronic data will be erased one year after collection and hard copies will be shredded after they are transcribed and analyzed.

The ethics protocol for this project (CUREB-B Clearance \# 107640)was reviewed by the Carleton University Research Ethics Board, which provided clearance to carry out the research. Should you have any ethical concerns with the study, please contact Dr. Andy Adler, Chair, Carleton University Research Ethics Board-B (by phone at $613-520-2600$ ext. 4085 or by email at ethics@carleton.ca).

Researchers contact information:

Yasin Farmani

School of Computer Science

Carleton University

yasinfarmani@cmail.carleton.ca

\section{Supervisor contact information:}

Dr. Robert Teather

School of Information Technology

Carleton University

613-520-2600×4176

rob.teather@carleton.ca

Do you agree to participate in this study? Yes No 


\section{B.2 Consent form for Translation Snapping technique}

\section{Garleten \\ Canada's Capital University \\ Consent Form}

Title: Snapping Viewpoint (Discrete Movement) as a Method of Reducing Cybersickness in Virtual Environment.

Date of ethics clearance: To be determined by the REB (as indicated on the clearance form)

Ethics Clearance for the Collection of Data Expires: To be determined by the REB (as indicated on the clearance form)

I choose to participate in a study on cybersickness (motion sickness). I acknowledge that this study aims to assess the strength and the weakness of using different methods of navigation for evaluating Cybersickness in VR. The researcher for this study is Master's student, Yasin Farmani. He is working under the supervision of Dr. Robert Teather in The School of Information Technology. The study should take approximately 30 minutes to complete. To be eligible, you must be an adult (over the age of 18) comfortable speaking English.

This study will be held in 2 sessions. It involves a pre-test questionnaire, an activity (20 minute VR exposure) and a post-test questionnaire (SSQ and Presence and discomfort score questionnaire). Some objective data (such as optimal path and your performance) will be calculated automatically with 3D software.

If you feel uncomfortable or nauseous, you have the right to end your participation in the study at any time during the session, for any reason. Simply tell the researcher that you want to end the session. If you withdraw from the study, all information you have provided will be immediately destroyed. We will only mention that one of the participant could not finish the test. You will receive $5 \$$ whether you finish the study or not. If you withdraw from the study in the second session you will receive $10 \$$ compensation.

All responses will be kept anonymous. All research data and notes will be kept on the researchers' password-protected computer. Research data will only be accessible by the researchers and the research supervisor. Once the project is completed, all research data will be securely destroyed. Electronic data will be

This document has been printed on both sides of a single sheet of paper. Please retain a copy of this document for your records. 


\section{Fing Carleton \\ Canada's Capital University}

erased one year after collection and hard copies will be shredded after they are transcribed and analyzed.

The ethics protocol for this project (CUREB-B Clearance \# 107640) was reviewed by the Carleton University Research Ethics Board, which provided clearance to carry out the research. If you have any ethical concerns with the study, please contact Dr. Andy Adler, Chair, Carleton University Research Ethics Board-A by phone at 613-520-2600 ext. 2517 or via email at ethics@carleton.ca.

\section{Researchers contact information:}

Yasin Farmani

School of Computer Science

Carleton University

yasinfarmani@cmail.carleton.ca

\section{Supervisor contact information:}

Dr. Robert Teather

School of Information Technology

Carleton University

613-520-2600x4176

rob.teather@carleton.ca

Signature of participant

Date

Signature of a researcher

Date

Page 2 of 2

This document has been printed on both sides of a single sheet of paper. Please retain a copy of this document for your records. 


\section{B.3 Simulator sickness questionnaire}

\begin{tabular}{|c|c|c|c|c|}
\hline No & \multicolumn{3}{|c|}{ Date } & \\
\hline \multicolumn{5}{|c|}{$\begin{array}{l}\text { SIMULATOR SICKNESS QUESTIONNAIRE } \\
\text { Kennedy, Lane, Berbaum, \& Lilienthal (1993)*** }\end{array}$} \\
\hline \multicolumn{5}{|c|}{ Instructions : Circle how much each symptom below is affecting you right now. } \\
\hline 1. General discomfort & $\underline{\text { None }}$ & $\underline{\text { Slight }}$ & $\underline{\text { Moderate }}$ & $\underline{\text { Severe }}$ \\
\hline 2. Fatigue & $\underline{\text { None }}$ & $\underline{\text { Slight }}$ & $\underline{\text { Moderate }}$ & $\underline{\text { Severe }}$ \\
\hline 3. Headache & $\underline{\text { None }}$ & $\underline{\text { Slight }}$ & $\underline{\text { Moderate }}$ & $\underline{\text { Severe }}$ \\
\hline 4. Eye strain & $\underline{\text { None }}$ & $\underline{\text { Slight }}$ & $\underline{\text { Moderate }}$ & $\underline{\text { Severe }}$ \\
\hline 5. Difficulty focusing & $\underline{\text { None }}$ & $\underline{\text { Slight }}$ & $\underline{\text { Moderate }}$ & $\underline{\text { Severe }}$ \\
\hline 6. Salivation increasing & $\underline{\text { None }}$ & $\underline{\text { Slight }}$ & $\underline{\text { Moderate }}$ & $\underline{\text { Severe }}$ \\
\hline 7. Sweating & None & $\underline{\text { Slight }}$ & $\underline{\text { Moderate }}$ & $\underline{\text { Severe }}$ \\
\hline 8. Nausea & $\underline{\text { None }}$ & $\underline{\text { Slight }}$ & $\underline{\text { Moderate }}$ & $\underline{\text { Severe }}$ \\
\hline 9. Difficulty concentrating & $\underline{\text { None }}$ & $\underline{\text { Slight }}$ & $\underline{\text { Moderate }}$ & $\underline{\text { Severe }}$ \\
\hline 10. « Fullness of the Head » & None & $\underline{\text { Slight }}$ & $\underline{\text { Moderate }}$ & $\underline{\text { Severe }}$ \\
\hline 11. Blurred vision & $\underline{\text { None }}$ & $\underline{\text { Slight }}$ & $\underline{\text { Moderate }}$ & $\underline{\text { Severe }}$ \\
\hline 12. Dizziness with eyes open & $\underline{\text { None }}$ & $\underline{\text { Slight }}$ & $\underline{\text { Moderate }}$ & $\underline{\text { Severe }}$ \\
\hline 13. Dizziness with eyes closed & $\underline{\text { None }}$ & $\underline{\text { Slight }}$ & Moderate & $\underline{\text { Severe }}$ \\
\hline 14. *Vertigo & None & $\underline{\text { Slight }}$ & Moderate & Severe \\
\hline 15. ${ }^{* *}$ Stomach awareness & $\underline{\text { None }}$ & $\underline{\text { Slight }}$ & Moderate & $\underline{\text { Severe }}$ \\
\hline 16. Burping & None & Slight & Moderate & $\underline{\text { Severe }}$ \\
\hline
\end{tabular}

Last version : March 2013

${ }^{* * *}$ Original version : Kennedy, R.S., Lane, N.E., Berbaum, K.S., \& Lilienthal, M.G. (1993). Simulator Sickness Questionnaire: An enhanced method for quantifying simulator sickness. International Journal of Aviation Psychology, $3(3), 203-220$. 


\section{B.4 Witmer and Singer Presence questionnaire}

\section{PRESENCE QUESTIONNAIRE}

(Witmer \& Singer, Vs. 3.0, Nov. 1994)*

Revised by the UQO Cyberpsychology Lab (2004)

Characterize your experience in the environment, by marking an " $\mathrm{X}$ " in the approp box of the 7-point scale, in accordance with the question content and descriptive la Please consider the entire scale when making your responses, as the intermediate 1 may apply. Answer the questions independently in the order that they appear. Dr skip questions or return to a previous question to change your answer.

\section{WITH REGARD TO THE EXPERIENCED ENVIRONMENT}

1. How much were you able to control events?

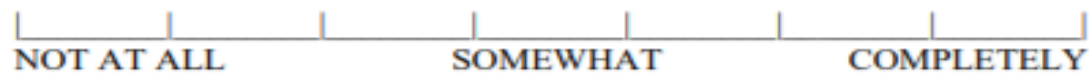

2. How responsive was the environment to actions that you initiated (or performed)?

\begin{tabular}{ll|l|} 
& $\mid$ & $\mid$ \\
NOT & MODERATELY & COMPLETELY \\
RESPONSIVE & RESPONSIVE & RESPONSIVE
\end{tabular}

3. How natural did your interactions with the environment seem?

\begin{tabular}{l|r|r|} 
& $\mid$ & $\mid$ \\
EXTREMELY & BORDERLINE & COMPLETELY \\
ARTIFICIAL & & NATURAL
\end{tabular}

4. How much did the visual aspects of the environment involve you?

\begin{tabular}{|l|l|l|}
$\mid$ & $\mid$ & $\mid$ \\
NOT AT ALL & SOMEWHAT & COMPLETELY
\end{tabular}

5. How natural was the mechanism which controlled movement through the environment?

\begin{tabular}{l|l|l|} 
& $\mid$ & $\mid$ \\
EXTREMELY & BORDERLINE & COMPLETELY \\
ARTIFICIAL & & NATURAL
\end{tabular}


6. How compelling was your sense of objects moving through space?

\begin{tabular}{|l|l|} 
NOT AT ALL & $\mid$ \\
MODERATELY & VERY \\
COMPELLING & COMPELLING
\end{tabular}

7. How much did your experiences in the virtual environment seem consistent with your real world experiences?

\begin{tabular}{llr|} 
& $\mid$ & $\mid$ \\
NOT & MODERATELY & VERY \\
CONSISTENT & CONSISTENT & CONSISTENT
\end{tabular}

8. Were you able to anticipate what would happen next in response to the actions that you performed?

\begin{tabular}{l|l|l|}
$\mid$ & $\mid$ & $\mid$ \\
NOT AT ALL & SOMEWHAT & $\mid$ \\
COMPLETELY
\end{tabular}

9. How completely were you able to actively survey or search the environment using vision?

\begin{tabular}{ll|l}
$\mid$ & $\mid$ & $\mid$ \\
NOT AT ALL & SOMEWHAT & $\mid$ \\
COMPLETELY
\end{tabular}

10. How compelling was your sense of moving around inside the virtual environment?

\begin{tabular}{llr|}
\hline NOT & $\mid$ & $\mid$ \\
COMPELLING & MODERATELY & VERY \\
COMPELLING & COMPELLING
\end{tabular}

11. How closely were you able to examine objects?

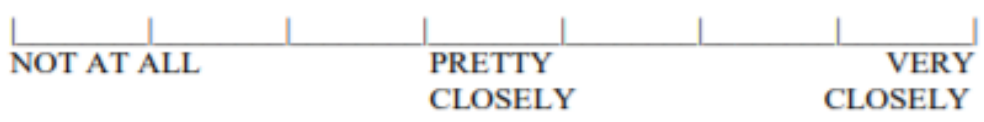

12. How well could you examine objects from multiple viewpoints?

\begin{tabular}{|l|l|l|}
$\mid$ & $\mid$ & $\mid$ \\
NOT AT ALL & SOMEWHAT & $\mid$ \\
\hline
\end{tabular}


IF THE VIRTUAL ENVIRONMENT INCLUDED SOUNDS:

20. How much did the auditory aspects of the environment involve you?

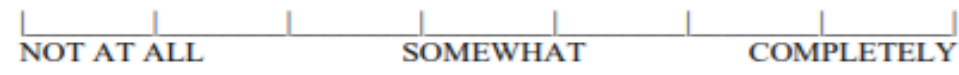

21. How well could you identify sounds?

\begin{tabular}{|l|l|l|}
$\mid$ & $\mid$ & $\mid$ \\
NOT AT ALL & SOMEWHAT & $\mid$ \\
COMPLETELY
\end{tabular}

22. How well could you localize sounds?

\begin{tabular}{l|l|l|}
$\mid$ & $\mid$ & $\mid$ \\
NOT AT ALL & SOMEWHAT & $\mid$
\end{tabular}

IF THE VIRTUAL ENVIRONMENT INCLUDED HAPTIC (SENSE OF TOUCH):

23. How well could you actively survey or search the virtual environment using touch?

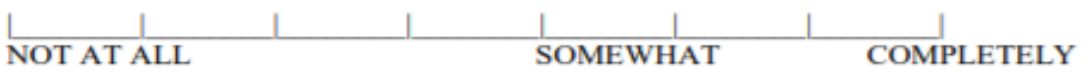

24. How well could you move or manipulate objects in the virtual environment?

\begin{tabular}{|l|l|}
$\mid$ & $\mid$ \\
NOT AT ALL SOMEWHAT & $\mid$ \\
EXTENSIVELY
\end{tabular}

Last version : March 2013

*Original version : Witmer, B.G. \& Singer. M.J. (1998). Measuring presence in virtual environments: A presence questionnaire. Presence : Teleoperators and Virtual Environments, 7(3), 225-240. Revised factor structure: Witmer, B.J., Jerome, C.J., \& Singer, M.J. (2005). The factor structure of the Presence Questionnaire. Presence, 14(3) 298-312. 
13. How involved were you in the virtual environment experience?

\begin{tabular}{l|cr|} 
& \multicolumn{1}{|c|}{$\mid$} & $\mid$ \\
NOT & MILDLY & COMPLETELY \\
INVOLVED & INVOLVED & ENGROSSED
\end{tabular}

14. How much delay did you experience between your actions and expected outcomes?

\begin{tabular}{|c|c|}
\hline NO DELAYS & $\mid$ \\
MODERATE \\
DELAYS
\end{tabular}

15. How quickly did you adjust to the virtual environment experience?

\begin{tabular}{l|l|l|}
$\mid$ & $\mid$ & $\mid$ \\
NOT AT ALL & SLOWLY THAN \\
ONE MINUTE
\end{tabular}

16. How proficient in moving and interacting with the virtual environment did you feel at the end of the experience?

\begin{tabular}{l|lr|} 
& $\mid$ & $\mid$ \\
NOT & REASONABLY & VERY \\
PROFICIENT & PROFICIENT & PROFICIENT
\end{tabular}

17. How much did the visual display quality interfere or distract you from performing assigned tasks or required activities?

\begin{tabular}{l|ll|}
$\mid$ & $\mid$ & $\mid$ \\
NOT AT ALL & INTERFERED & PREVENTED \\
& SOMEWHAT & TASK PERFORMANCE
\end{tabular}

18. How much did the control devices interfere with the performance of assigned tasks or with other activities?

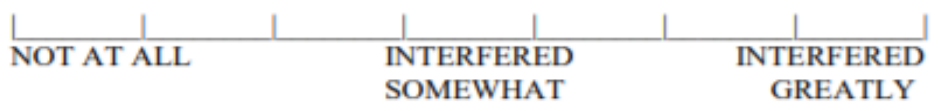

19. How well could you concentrate on the assigned tasks or required activities rather than on the mechanisms used to perform those tasks or activities?

\begin{tabular}{l|l|l|}
$\mid$ & $\mid$ & $\mid$ \\
NOT AT ALL & SOMEWHAT & $\mid$ \\
COMPLETELY
\end{tabular}




\section{B.5 NASA TLX Questionnaire}

\section{NASA Task Load Index}

Hart and Staveland's NASA Task Load Index (TLX) method assesses work load on five 7-point scales. Increments of high, medium and low estimates for each point result in 21 gradations on the scales.

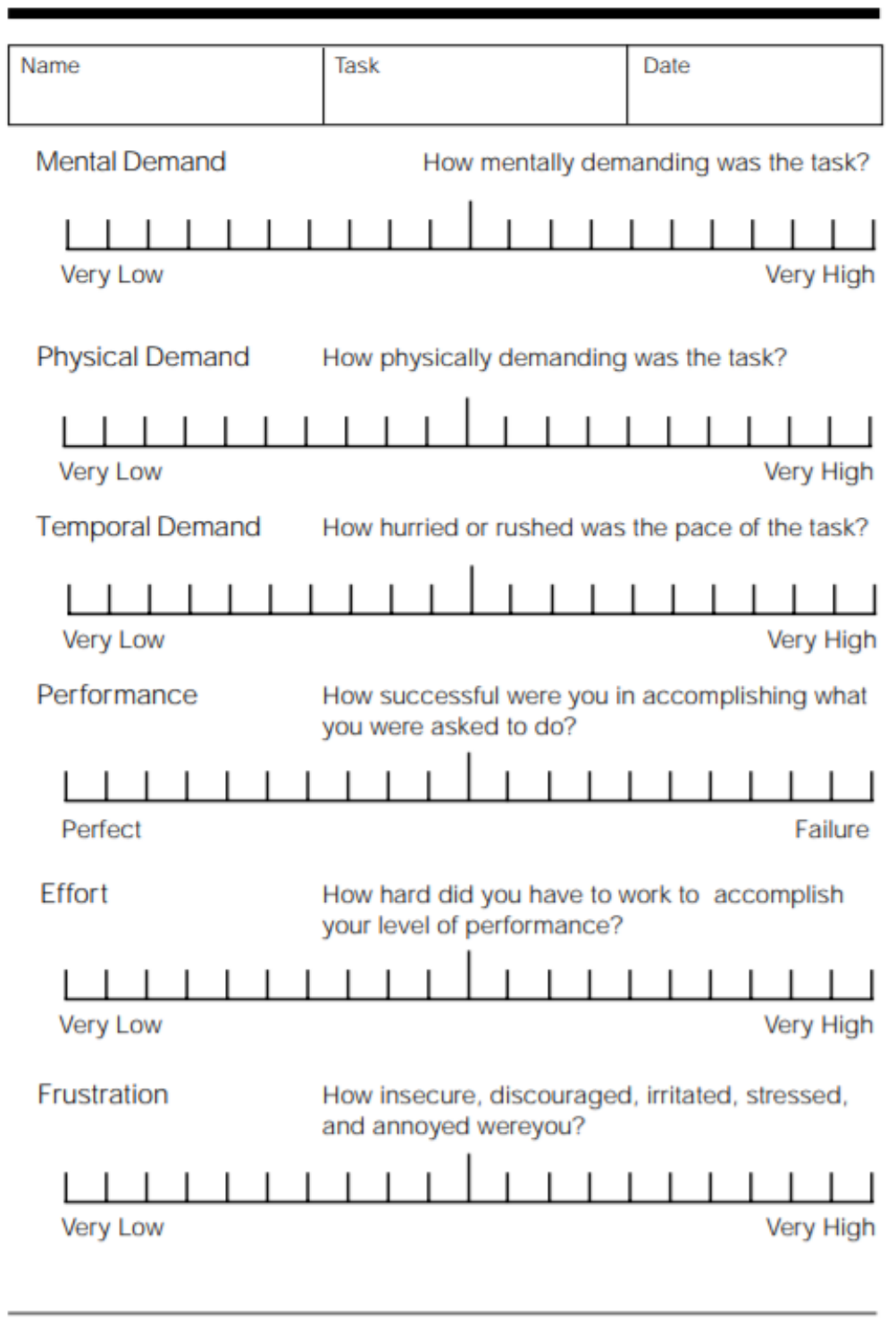




\section{B.6 Demographic form}

Discrete Movement and Translation as a Method for Reducing Cybersickness in Virtual Environments?

Demographics

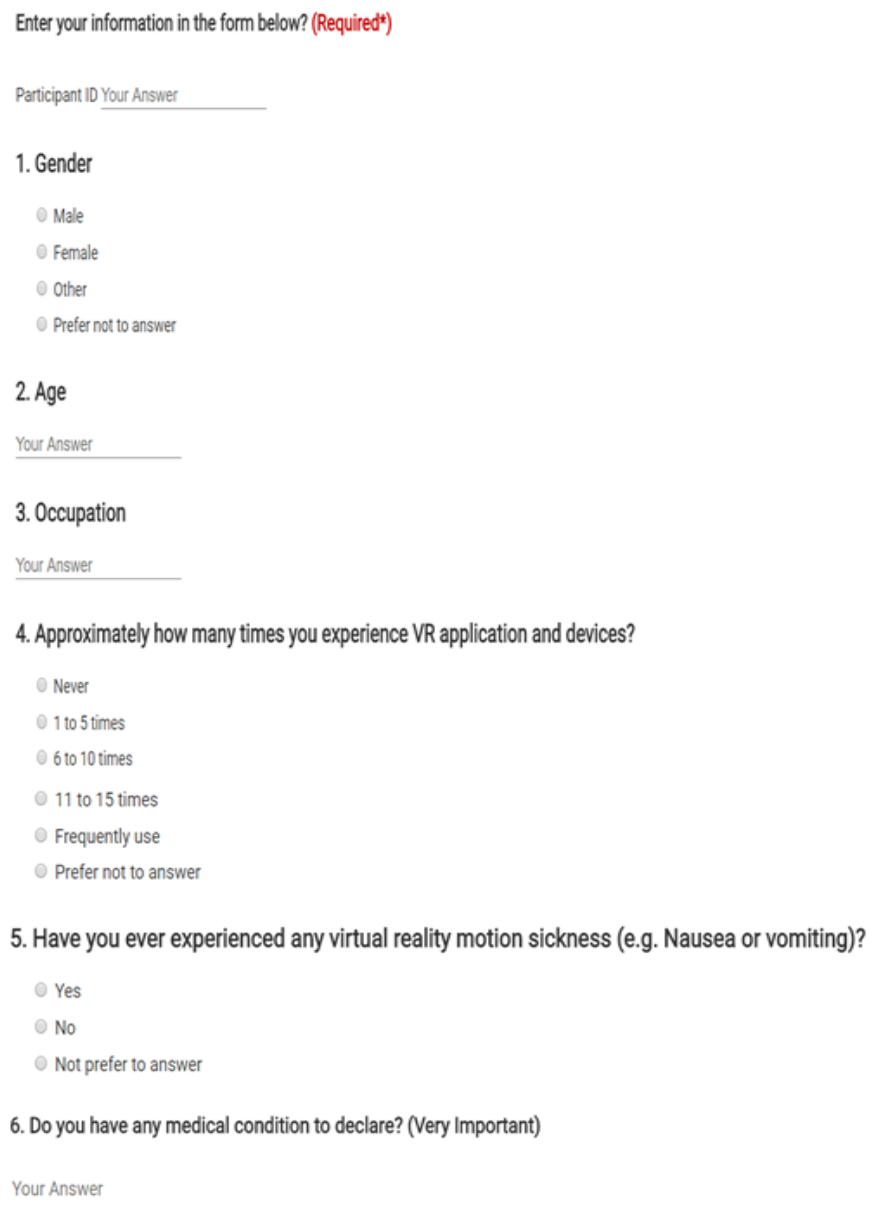




\section{References}

[1] Arns, L.L. and Cerney, M.M. 2005. The relationship between age and incidence of cybersickness among immersive environment users. IEEE Proceedings, Virtual Reality. (2005), 267-268. DOI:https://doi.org/10.1109/VR.2005.1492788.

[2] Bhandari, Jiwan and MacNeilage, Paul and Folmer, E. 2018. Teleportation without Spatial Disorientation Using Optical Flow Cues. Graphics interface (Toronto, Ontario, 2018), 153-158.

[3] Bonato, F., Bubka, A., Palmisano, S., Phillip, D. and Moreno, G. 2008. Vection Change Exacerbates Simulator Sickness in Virtual Environments. Presence: Teleoperators and Virtual Environments. 17, 3 (Jun. 2008), 283-292. DOI:https://doi.org/10.1162/pres.17.3.283.

[4] Bowman, D.A., Koller, D. and Hodges, L.F. 1997. Travel in Immersive Virtual Environments : An Evaluation of Viewpoint Motion Control Techniques Georgia Institute of Technology. Proceedings of the 1997 Virtual Reality Annual International Symposium. $\quad$ (1997), DOI:https://doi.org/10.1109/VRAIS.1997.583043.

[5] Bowman, D. a, Mcmahan, R.P. and Tech, V. 2007. Virtual Reality: How Much Immersion Is Enough? (Cover story). Computer. 40, 7 (2007), 36-43. DOI:https://doi.org/10.1109/MC.2007.257.

[6] Brand, J.T.R. and J.J. 1975. Motion Sickness. London: Academic press.

[7] Budhiraja, P., Miller, M.R., Modi, A.K. and Forsyth, D. 2017. Rotation Blurring: 
Use of Artificial Blurring to Reduce Cybersickness in Virtual Reality First Person Shooters. (Oct. 2017).

[8] Chang, C.-H., Pan, W.-W., Chen, F.-C. and Stoffregen, T.A. 2013. Console video games, postural activity, and motion sickness during passive restraint. Experimental Brain Research. 229, 2 (Aug. 2013), 235-242. DOI:https://doi.org/10.1007/s00221013-3609-y.

[9] Chang, E., Hwang, I., Jeon, H., Chun, Y., Kim, H.T. and Park, C. 2013. Effects of rest frames on cybersickness and oscillatory brain activity. 2013 International Winter Workshop on Brain-Computer Interface (BCI) (Feb. 2013), 62-64.

[10] Chen, D.J. 2014. Frequncy Responses of Visually Induced Motion Sickness: Isolating Effects of Velocity and Amplitude of Visual Stimuli. Hong Kong.

[11] Cloudstep - Zero Vection VR Locomotion Thingy - YouTube: https://www.youtube.com $/$ watch? $v=v V V d o q u K h O 8 \& t=15$ s. Accessed: 2018-01-29.

[12] Davis, S., Nesbitt, K. and Nalivaiko, E. 2014. A Systematic Review of Cybersickness. Proceedings of the 2014 Conference on Interactive Entertainment IE2014. (2014), 1-9. DOI:https://doi.org/10.1145/2677758.2677780.

[13] Davis, S., Nesbitt, K. and Nalivaiko, E. 2015. Comparing the onset of cybersickness using the Oculus Rift and two virtual roller coasters. 11th Australasian Conference on Interactive Entertainment (IE 2015). January (2015), 27-30. DOI:https://doi.org/10.17973/MMSJ.2015.

[14] Dennison, M.S., Wisti, A.Z. and D'Zmura, M. 2016. Use of physiological signals to 
predict $\quad$ cybersickness. $\quad$ Displays. $\quad 44, \quad$ (2016), $\quad 42-52$. DOI:https://doi.org/10.1016/j.displa.2016.07.002.

[15] Dorado, J.L. and Figueroa, P.A. 2014. Ramps are better than stairs to reduce cybersickness in applications based on a HMD and a Gamepad. IEEE Symposium on $3 D$ User Interfaces 2014, 3DUI 2014 - Proceedings. (2014), 47-50. DOI:https://doi.org/10.1109/3DUI.2014.6798841.

[16] Eugenia M. Kolasinski and Richard D. Gilson 1998. Simulator sickness and related findings in a virtual reality. PROCEEDINGS of the HUMAN FACTORS AND ERGONOMICS SOCIETY 42nd ANNUAL MEETING. (1998).

[17] Farmani, Yasin and Teather, R. 2018. Viewpoint Snapping to Reduce Cybersickness in Virtual Reality. Graphics interface (Toronto, Ontario, 2018), 159-166.

[18] Farmani, Y. and Teather, R.J. 2017. Player Performance with Different Input Devices in Virtual Reality First-Person Shooter Games. 1, (2017). DOI:https://doi.org/10.1145/3131277.3134361.

[19] Fernandes, A.S. and Feiner, S.K. 2016. Combating VR sickness through subtle dynamic field-of-view modification. 2016 IEEE Symposium on 3D User Interfaces, $3 D U I \quad 2016 \quad-\quad$ Proceedings. $\quad$ (2016), 201-210. DOI:https://doi.org/10.1109/3DUI.2016.7460053.

[20] Hart, S.G. 2006. Nasa-Task Load Index (NASA-TLX); 20 Years Later. Proceedings of the Human Factors and Ergonomics Society Annual Meeting. 50, 9 (Oct. 2006), 904-908. DOI:https://doi.org/10.1177/154193120605000909. 
[21] Hecht, J. 2016. Optical Dreams, Virtual Reality. Optics and Photonics News. 27, 6 (Jun. 2016), 24. DOI:https://doi.org/10.1364/OPN.27.6.000024.

[22] Hettinger, L.J., Berbaum, K.S., Kennedy, R.S., Dunlap, W.P. and Nolan, M.D. 1990. Vection and simulator sickness. Military Psychology. 2, 3 (1990), 171-181. DOI:https://doi.org/10.1207/s15327876mp0203_4.

[23] Hettinger, L.J. and Riccio, G.E. 1992. Visually Induced Motion Sickness in Virtual Environments. Presence: Teleoperators and Virtual Environments. 1, 3 (Jan. 1992), 306-310. DOI:https://doi.org/10.1162/pres.1992.1.3.306.

[24] Hu, S., McChesney, K., Player, K., Aviation, A.B.-, space, undefined, and, undefined and 1999, undefined Systematic investigation of physiological correlates of motion sickness induced by viewing an optokinetic rotating drum. psycnet.apa.org.

[25] HU, S., STERN, R., ... M.V.- and 1988, undefined MOTION SICKNESS AND ELECTROGASTROGRAPHIC ACTIVITY AS A FUNCTION OF SPEED OF ROTATION OF AN OPTOKINETIC DRUM. SOC PSYCHOPHYSIOL RES $1010 \ldots$

[26] JF, G. 1998. Motion Sickness Susceptibility Questionnaire Short-form (MSSQShort). Brain Research Bulletin,. 47, (1998), 507-516.

[27] Johnson, D.M. 2005. Introduction to and Review of Simulator Sickness Research.

[28] Kellogg, R., Kennedy, R. and Graybiel, A. 1964. Motion sickness symptomatology of labyrinthine defective and normal subjects during zero gravity maneuvers. 
(1964).

[29] Kemeny, A. and George, P, F.M.- 2017. New VR Navigation Techniques to Reduce Cybersickness. ingentaconnect.com - Electronic. (2017).

[30] Kennedy, R., Hettinger, L., ... D.H.-J. of and 1996, undefined Psychophysical scaling of circular vection $(\mathrm{CV})$ produced by optokinetic $(\mathrm{OKN})$ motion: individual differences and effects of practice. content.iospress.com.

[31] Kennedy, R.S., Lane, N.E., Berbaum, K.S. and Lilienthal, M.G. 1993. Simulator Sickness Questionnaire: An Enhanced Method for Quantifying Simulator Sickness. The International Journal of Aviation Psychology. 3, 3 (1993), 203-220. DOI:https://doi.org/10.1207/s15327108ijap0303_3.

[32] Kennedy, R.S., Stanney, K.M. and Dunlap, W.P. 2000. Duration and Exposure to Virtual Environments: Sickness Curves During and Across Sessions. Presence: Teleoperators and Virtual Environments. 9, 5 (Oct. 2000), 463-472. DOI:https://doi.org/10.1162/105474600566952.

[33] Keshavarz, B. and Hecht, H. 2011. Axis rotation and visually induced motion sickness: The role of combined roll, pitch, and yaw motion. Aviation Space and $\begin{array}{lllll}\text { Environmental } \quad \text { Medicine. } & \text { 82, } & 11 & \text { (2011), } & \text { 1023-1029. }\end{array}$ DOI:https://doi.org/10.3357/ASEM.3078.2011.

[34] Keshavarz, B., Riecke, B.E., Hettinger, L.J. and Campos, J.L. 2015. Vection and visually induced motion sickness: How are they related? Frontiers in Psychology. 6, APR (2015), 1-11. DOI:https://doi.org/10.3389/fpsyg.2015.00472. 
[35] Keshavarz, B., Stelzmann, D., Paillard, A. and Hecht, H. 2015. Visually induced motion sickness can be alleviated by pleasant odors. Experimental Brain Research. 233, 5 (2015), 1353-1364. DOI:https://doi.org/10.1007/s00221-015-4209-9.

[36] Kim, Y.Y., Kim, H.J., Kim, E.N., Ko, H.D. and Kim, H.T. 2005. Characteristic changes in the physiological components of cybersickness. Psychophysiology. 42, 5 (Aug. 2005), 616-625. DOI:https://doi.org/10.1007/s00234-005-1388-2.

[37] LaViola, J.J. 2000. A discussion of cybersickness in virtual environments. $A C M$ SIGCHI Bulletin. 32, 1 (2000), 47-56. DOI:https://doi.org/10.1145/333329.333344.

[38] Liu, L., Miyazaki, M. and Watson, B. 1999. Norms and validyt of the DriVR: A virtual reality driving assessment for persons with head injuries. CyberPsychology \& Behavior. Vol 2(1). (1999), 53-67. DOI:https://doi.org/10.1089/cpb.1999.2.53 10.1089/cpb.1999.2.53 [pii].

[39] Lo, W., ergonomics, R.S.-A. and 2001, undefined Cybersickness in the presence of scene rotational movements along different axes. Elsevier.

[40] Loomis, J., Klatzky, R., ... R.G.-J. of and 1993, undefined Nonvisual navigation by blind and sighted: assessment of path integration ability. psycnet.apa.org.

[41] McCauley, M.E. and Sharkey, T.J. 1992. Cybersickness: Perception of Self-Motion in Virtual Environments. Presence: Teleoperators and Virtual Environments. 1, 3 (Jan. 1992), 311-318. DOI:https://doi.org/10.1162/pres.1992.1.3.311.

[42] McCauley, M.E. and Sharkey, T.J. 1992. Cybersickness: Perception of Self-Motion in Virtual Environments. Presence: Teleoperators and Virtual Environments. 1, 3 
(Jan. 1992), 311-318. DOI:https://doi.org/10.1162/pres.1992.1.3.311.

[43] Park, G.D. et al. 2014. Motion sickness adaptation: a neural mismatch model. Human factors. 43, 1 (2014), 47-50. DOI:https://doi.org/10.1007/s00371-008$0277-1$.

[44] Park, G.D., Allen, R.W., Fiorentino, D., Rosenthal, T.J. and Cook, M.L. 2006. Simulator Sickness Scores According to Symptom Susceptibility, Age, and Gender for an Older Driver Assessment Study. Proceedings of the Human Factors and Ergonomics Society Annual Meeting. 50, 26 (2006), 2702-2706. DOI:https://doi.org/10.1177/154193120605002607.

[45] R.Yao, T. Heath, A. Davies, T. Forsyth, N. Mitchell, and P.H. 2016. Oculus Best Practices. Oculus Documentation. (2016), Introduction to Best Practices.

[46] Rebenitsch, L. and Owen, C. 2016. Review on cybersickness in applications and visual displays. Virtual Reality. 20, 2 (2016), 101-125. DOI:https://doi.org/10.1007/s10055-016-0285-9.

[47] Regan, C. 1995. An investigation into nausea and other side-effects of head-coupled immersive virtual reality. Virtual Reality. 1, 1 (Jun. 1995), 17-31. DOI:https://doi.org/10.1007/BF02009710.

[48] Regan, C. 1995. An investigation into nausea and other side-effects of head-coupled immersive virtual reality. Virtual Reality. 1, 1 (Jun. 1995), 17-31. DOI:https://doi.org/10.1007/BF02009710.

[49] Riccio, G.E. and Stoffregen, T.A. 1991. An Ecological Theory of Motion Sickness 
and Postural Instability. Ecological Psychology.

[50] Ryge, Andreas Nicolaj, Casper Gade Kranker Vollmers, Jonatan Salling Hvass, Lars Koreska Andersen, Jon Ram Bruun-Pedersen, Niels Chr. Nilsson, and R.N. 2018. A Preliminary Investigation of the Effects of Discrete Virtual Rotation on Cybersickness. Virtual Reality (vr), 2018 (2018).

[51] Sanchez-Vives, M. V. and Slater, M. 2005. Opinion: From presence to consciousness through virtual reality. Nature Reviews Neuroscience. 6, 4 (Apr. 2005), 332-339. DOI:https://doi.org/10.1038/nrn1651.

[52] Seay, A.F., Krum, D.M., Hodges, L. and Ribarsky, W. Simulator sickness and presence in a high FOV virtual environment. Proceedings IEEE Virtual Reality 2001 299-300.

[53] Seno, T., Ito, H. and Sunaga, S. 2011. Inconsistent Locomotion Inhibits Vection. Perception. 40, 6 (Jun. 2011), 747-750. DOI:https://doi.org/10.1068/p7018.

[54] Sharples, S., Cobb, S., Moody, A., Displays, J.W.- and 2008, undefined Virtual reality induced symptoms and effects (VRISE): Comparison of head mounted display (HMD), desktop and projection display systems. Elsevier.

[55] So, R.H., Lo, W.T. and Ho, a T. 2001. Effects of navigation speed on motion sickness caused by an immersive virtual environment. Human factors. 43, 3 (2001), 452-461. DOI:https://doi.org/10.1518/001872001775898223.

[56] Stanney, K.M. and Kennedy, R.S. 1997. The Psychometrics of Cybersickness. Communications of the ACM. 40, 8 (1997), 67-68. 
[57] Stanney, K.M., Kennedy, R.S. and Drexler, J.M. 1997. Cybersickness is Not Simulator Sickness. Proceedings of the Human Factors and Ergonomics Society Annual Meeting. 41, 2 (Oct. 1997), 1138-1142. DOI:https://doi.org/10.1177/107118139704100292.

[58] Stanney, K.M., Kennedy, R.S. and Drexler, J.M. 1997. Cybersickness is Not Simulator Sickness. Proceedings of the Human Factors and Ergonomics Society Annual Meeting. 41, 2 (Oct. 1997), 1138-1142. DOI:https://doi.org/10.1177/107118139704100292.

[59] Treisman, M. 1977. Motion Sickness: An Evolutionary Hypothesis. Science. 197, 4302 (1977), 493-495. DOI:https://doi.org/10.1126/science.301659.

[60] Trutoiu, L., Mohler, B., ... J.S.-P.-C.\& and 2009, undefined Circular, linear, and curvilinear vection in a large-screen virtual environment with floor projection. Elsevier.

[61] Tschermak, A. 1931. Optischer Raumsinn. Receptionsorgane II. Springer Berlin Heidelberg. 834-1000.

[62] VR Bits - Making VR Bit by Bit: http://www.vr-bits.com/. Accessed: 2018-01-29.

[63] WeiBker, T., Bernd, K., Ohlich, F. and Kulik, A. 2018. Spatial Updating and Simulator Sickness during Steering and Jumping in Immersive Virtual Environments. IEEE VR (2018), 256-307.

[64] Witmer, B.G. and Singer, M.J. 1998. Measuring Presence in Virtual Environments: A Presence Questionnaire. Presence: Teleoper. Virtual Environ. 7, 3 (1998), 225- 
240. DOI:https://doi.org/10.1162/105474698565686. 